\title{
Cohomological reduction of sigma models
}

\author{
Constantin Candu, ${ }^{a}$ Thomas Creutzig, ${ }^{b}$ Vladimir Mitev ${ }^{a}$ and Volker Schomerus ${ }^{a}$ \\ ${ }^{a}$ DESY Hamburg, Theory Group, \\ Notkestrasse 85, D-22607 Hamburg, Germany \\ ${ }^{b}$ Department of Physics and Astronomy, University of North Carolina, \\ Phillips Hall, CB 3255 Chapel Hill, NC 27599-3255, U.S.A. \\ E-mail: Constantin.Candu@desy.de, creutzig@physics.unc.edu, \\ Vladimir.Mitev@desy.de, Volker.Schomerus@desy.de
}

ABSTRACT: This article studies some features of quantum field theories with internal supersymmetry, focusing mainly on 2-dimensional non-linear sigma models which take values in a coset superspace. It is discussed how BRST operators from the target space supersymmetry algebra can be used to identify subsectors which are often simpler than the original model and may allow for an explicit computation of correlation functions. After an extensive discussion of the general reduction scheme, we present a number of interesting examples, including symmetric superspaces $G / G^{\mathbb{Z}_{2}}$ and coset superspaces of the form $G / G^{\mathbb{Z}_{4}}$.

Keywords: Sigma Models, Superspaces, Topological Field Theories, Global Symmetries

ARXIV EPRINT: 1001.1344 


\section{Contents}

1 Introduction 1

2 Sigma models on coset superspaces $G / G^{\prime} \quad 4$

2.1 General coset superspaces $G / G^{\prime}$

$2.2 G / G^{\mathbb{Z}_{N}}$ coset superspaces 6

2.3 Observables and correlators 8

3 Cohomological reduction in representation theory 9

3.1 Overview over results 9

$\begin{array}{ll}3.2 & \text { Reduction of Lie superalgebras } \\ 3.32\end{array}$

$\begin{array}{lll}3.3 & \text { Reduction of modules } & 19\end{array}$

3.4 Reduction of smooth functions on $G / G^{\prime} \quad 22$

3.5 Reduction of smooth tensor forms on $G / G^{\prime} \quad 25$

$\begin{array}{ll}3.6 & \text { Reduction of } L_{2}\left(G / G^{\prime}\right) \\ 25\end{array}$

4 Cohomological reduction in the field theory $\quad 27$

5 Applications $\quad 28$

$\begin{array}{ll}5.1 & \text { Conformal field theory } \\ 5.28\end{array}$

$\begin{array}{ll}5.2 \text { Sigma models on symmetric superspaces } & 29\end{array}$

$\begin{array}{lll}5.3 & \text { Examples involving generalized symmetric spaces } & 31\end{array}$

5.4 Extensions of the cohomological reduction 34

6 Conclusion and outlook $\quad 35$

\section{Introduction}

Due to their appearance in many quite distinct areas of physics, non-linear sigma models with target space (i.e. internal) supersymmetry have been the subject of much interest lately. One area in which they appear are the proposed dualities between string theories in Anti de Sitter backgrounds $A d S_{n+1} \times M$ and conformal gauge theories, the most well known examples being $A d S_{5} \times S^{5}$ and $A d S_{4} \times \mathbb{C P}^{3}$ which are described e.g. in [1-6]. Other lines of applications involve dense polymers in two dimensions $[7,8]$, the quantum Hall plateau transitions [9] or disordered electron systems [10].

Sigma models on target superspaces possess a number of surprising properties which are gradually being uncovered. In particular, there exists several basic series of models which give rise to families of conformal field theories with continuously varying exponents, 
including the supergroup manifolds PSL $((|() \mathrm{N}| \mathrm{N}), \operatorname{OSP}(2 \mathrm{~N}+2 \mid 2 \mathrm{~N})$ and a number of quotients thereof $[9,11-14]$. Note that quantum conformal symmetry does not require the addition of any Wess-Zumino term, in contrast to the case of purely bosonic target spaces.

Solving conformal field theories with continuously varying exponents requires developing entirely new techniques which go far beyond the conventional algebraic methods. Numerical and algebraic studies of lattice discretizations $[13,15,16]$ and supersymmetry aided all-order perturbative computations of spectra [17, 18] have been applied with astonishing results. In some cases is was possible to determine exact formulas for all (boundary) conformal weights as a function of the continuous couplings (moduli) of the models.

Having gained some control over the weights it is a natural next step to investigate properties of higher correlation functions. While general correlators seem way out of reach, we will be able to gain useful insights into correlation functions involving a special subset of fields. Some inspiration can be taken from the study of conformal field theories with $N=(2,2)$ world-sheet supersymmetry. For such models, a very conventional trick that one exploits through the so-called topological twists, is to identify special subsectors whose dependence on the couplings can be brought under control. The idea is to employ a fermionic world-sheet symmetry generator as a BRST operator and to select its cohomology as the relevant subsector. If the action of the model is trivial in cohomology the correlation functions of subsector operators do not depend on the coupling constants of the theory. Such correlators can then be calculated in the (classical) limit, as described for example in [19].

The models we are interested in possess target space rather than world-sheet supersymmetry. A natural idea then is to promote an internal nilpotent symmetry to a BRST operator. In following this lead, we shall uncover a rather remarkable structure. Suppose we are starting with a sigma model on the quotient $G / G^{\prime}$, defined as the set of right $G^{\prime}$ cosets in $G$, with $G^{\prime}$ being some sub-supergroup of the supergroup $G$. Let $Q$ be some fermionic generator in the superalgebra $\mathfrak{g}^{\prime} \subset \mathfrak{g}$ such that $Q^{2}=0$. Note that such a $Q$ is a symmetry of the $G / G^{\prime}$ sigma model. Through its cohomology, $Q$ defines a subsector. Quite remarkably, the latter turns out to form the state space of another sigma model on the coset superspace $H / H^{\prime}$ with a new pair of supergroups $H^{\prime} \subset H$. The target space $H / H^{\prime}$ has smaller dimension than $G / G^{\prime}$ and the symmetry algebra $\mathfrak{h}$ of the reduced theory is contained in the symmetry algebra $\mathfrak{g}$. In many cases, further reduction is possible until the procedure terminates because the remaining symmetry algebra does not contain any further nilpotent generators. Thereby, we obtain a chain of models $\left\{\mathcal{M}_{\alpha}\right\}_{\alpha \in A}$ which is parametrized by elements $\alpha$ of some partially ordered set $A$. The model $\mathcal{M}_{\alpha}$ is a subsector of $\mathcal{M}_{\beta}$, i.e. $\mathcal{M}_{\alpha} \subset \mathcal{M}_{\beta}$, whenever $\alpha<\beta$. Let us give just one example here. It is provided by the following family of symmetric superspaces

$$
\mathcal{M}_{\left(\alpha_{1}, \alpha_{2}\right)}^{\mathrm{U} / \mathrm{U}^{2}}(R, S) \cong \frac{\mathrm{U}\left(R+S+\alpha_{1}+\alpha_{2} \mid S+\alpha_{1}+\alpha_{2}\right)}{\mathrm{U}\left(R+S+\alpha_{1} \mid \alpha_{1}\right) \times \mathrm{U}\left(\alpha_{2} \mid S+\alpha_{2}\right)}
$$

where $R, S, \alpha_{1}$ and $\alpha_{2}$ are all taken to be non-negative integers. The family (1.1) includes the complex projective spaces $\mathbb{C P}^{\mathrm{R}+\alpha_{1} \mid \alpha_{1}+1}$ for $S=1$ and $\alpha_{2}=0$.

In order to select conformal quotients from the list (1.1), we note that a theory can only be conformally invariant if all of its subsectors are. The smallest subsector in these families 
is obtained for $\alpha_{1}=\alpha_{2}=0$ and takes the simple form $\mathrm{U}(R+S \mid S) / \mathrm{U}(R+S) \times \mathrm{U}(S)$, which is a purely fermionic theory. For $R=0$ and $S=1$, this subsector is the theory of free symplectic fermions, but in all other cases it is a theory of interacting symplectic fermions, which, unsurprisingly, is massive. Hence the only candidates for conformal quotients one can find within the list (1.1) are of the form

$$
\mathcal{C}_{\left(\alpha_{1}, \alpha_{2}\right)}^{\mathrm{U} / \mathrm{U}^{2}}: \cong \mathcal{M}_{\left(\alpha_{1}, \alpha_{2}\right)}^{\mathrm{U} / \mathrm{U}^{2}}(0,1) \cong \frac{\mathrm{U}\left(\alpha_{1}+\alpha_{2}+1 \mid \alpha_{1}+\alpha_{2}+1\right)}{\mathrm{U}\left(1+\alpha_{1} \mid \alpha_{1}\right) \times \mathrm{U}\left(\alpha_{2} \mid 1+\alpha_{2}\right)}
$$

with $\alpha_{1}, \alpha_{2} \geq 0$. We thus observe that the technique of cohomological reduction provides more stringent constraints on possible candidate target spaces for conformally invariant sigma models than the computation of the first order $\beta$ function, which in this case, as was argued for instance in [14], requires only $R=0$. A priori, we cannot say for sure whether the spaces in (1.2) lead to conformal sigma models, just that, of all the spaces in (1.1), they are the only possible candidates. We shall however argue in subsection 5.1 that symmetric spaces that possess a non-trivial conformal subsector with non-zero central charge are actually conformal. This is due to the fact ${ }^{1}$ that for symmetric spaces, the metric and 2-form determining the model are fixed by the symmetry up to a prefactor. Since the theory of free symplectic fermions has central charge $c=-2 \neq 0$, all the models in the list (1.2) give rise to conformal sigma models. The list includes the complex projective superspaces $\mathbb{C P}^{\alpha_{1} \mid \alpha_{1}+1}$ for which conformal invariance has been established before (see e.g. $[13,21]$ ). We shall extend this discussion to arbitrary compact symmetric superspaces in section 5.1. Within this class, we shall thereby recover the complete classification of conformal models from [22], which was obtained by analyzing the all order $\beta$ function. $^{2}$

But our approach is more general. It also applies to all coset superspaces $G / G^{\prime}$ without any additional assumption on the denominator subgroup $G^{\prime}$. In section 5.2 we look at examples for which $G^{\prime}$ is fixed under the action of some automorphism of order four, i.e. at quotients of the form $G / G^{\mathbb{Z}_{4}}$. Such generalized symmetric spaces have become popular through the investigation of strings in Anti de Sitter backgrounds. While we are not aiming at an exhaustive investigation of quotients within this class, we shall exhibit a few interesting examples, including the family

$$
\mathcal{M}_{\left(\alpha_{1}, \alpha_{2}\right)}^{\mathrm{U} / \mathrm{OSP}^{2}}(S) \cong \frac{\operatorname{PSU}\left(2 S+2 \alpha_{1}+2 \alpha_{2} \mid 2 S+2 \alpha_{1}+2 \alpha_{2}\right)}{\operatorname{OSP}\left(2 S+2 \alpha_{1} \mid 2 \alpha_{1}\right) \times \operatorname{OSP}\left(2 S+2 \alpha_{2} \mid 2 \alpha_{2}\right)}
$$

with some obvious restrictions on the choice of $\alpha_{i}$ and $S$ such that all supergroups are welldefined. Note that, provided the $\alpha_{i}$ are large enough, the parameter $S$ may now assume any integer value, i.e. it can also be negative. The minimal non-trivial subsector of these theories depends significantly on the parameter $S$. It is given by

$$
\begin{array}{ll}
\mathcal{R}^{\mathrm{PSU} / \mathrm{OSP}^{2}}(S) \cong \frac{\mathrm{PSU}(2 \mathrm{~S} \mid 2 \mathrm{~S})}{\mathrm{SO}(() 2 \mathrm{~S}) \times \mathrm{SO}(() 2 \mathrm{~S})} & \text { for } S>0 \\
\mathcal{R}^{\mathrm{PSU} / \mathrm{OSP}^{2}}(0) \cong \text { symplectic fermions } & \text { for } S=0
\end{array}
$$

\footnotetext{
${ }^{1}$ See for instance [20].

${ }^{2}$ It turns out that for symmetric superspaces the first and second order are already enough to determine conformal invariance.
} 


$$
\mathcal{R}^{\mathrm{PSU} / \mathrm{OSP}^{2}}(S) \cong \frac{\mathrm{PSU}(-2 \mathrm{~S} \mid-2 \mathrm{~S})}{\mathrm{SP}(-2 \mathrm{~S}) \times \mathrm{SP}(-2 \mathrm{~S})} \quad \text { for } \quad S<0
$$

These are not conformal for $S \neq 0$ and reduce to a free theory for $S=0$. The smallest interacting theory for $S=0$ is obtained for $\alpha_{1}=1, \alpha_{2}=0$ and is the complex projective superspace

$$
\frac{\operatorname{PSU}(2 \mid 2)}{\operatorname{OSP}(2 \mid 2)} \cong \mathbb{C P}^{1 \mid 2}
$$

For higher values of $\alpha_{i}$ however, the superspaces are not of the complex projective type. It would be interesting to understand whether the family (1.3) with $S=0$ is conformally invariant. We have little more to say about this issue for now.

The series (1.3) contains a few other interesting minimal subsectors. In fact, for the $S=1$, the minimal subsector is given in eq. (1.4). After an appropriate change in the choice of reality conditions, we obtain the coset geometry for $A d S_{2} \times S^{2}$ as defined in [23]. Similarly, if we set $S=-2$ and perform again the appropriate change of the real form, we find the quotient that appears in the description of $A d S_{5} \times S^{5}$. Throughout most of this text, we shall consider sigma models without Wess-Zumino terms, mostly in order not to clutter the presentation too much. We shall comment on the possible inclusion of Wess-Zumino terms and the application to other 2-dimensional field theories in the concluding section.

We finish this introduction with a short guide for the subsequent sections. In the next section 2 we shall set the stage by defining in detail the models that we are going to consider. Subsections 3.2 to 3.6 then present the main mathematical tools at our disposal. Since these parts are a bit technical, we included a non-technical summary in subsection 3.1. The impatient reader may therefore skip subsections 3.2 to 3.6, at least upon first reading. The mathematical background from section 3 is then used in section 4 to prove the main results of this work. In section 5 we shall illustrate how the cohomological reduction works for symmetric superspaces. Once this is understood, we venture into generalized symmetric spaces. Our conclusion contains a few more comments on possible applications to more types of models and to AdS backgrounds in string theory.

\section{Sigma models on coset superspaces $G / G^{\prime}$}

The purpose of this section is to set the stage for our subsequent investigation. We shall provide two different formulations for non-linear sigma models on a right-coset superspace of the form $G / G^{\prime}$. Here $G$ is some supergroup with non-degenerate metric and $G^{\prime}$ is a sub-supergroup. For the moment, no further assumption is made concerning the structure of $G^{\prime}$. In later sections, fermionic elements of $G^{\prime}$ shall play a key role. There exist some tricks to extend the validity of our analysis in case $G^{\prime}$ does not contain any such elements. We shall discuss these briefly in case $G^{\prime}=\{e\}$ is trivial. The examples in section 5 focus on models in which $G^{\prime}=G^{\mathbb{Z}_{n}}$ is invariant under some automorphism of order $n=2$ or $n=4$. But for the general framework such special features of $G^{\prime}$ are irrelevant. 


\subsection{General coset superspaces $G / G^{\prime}$}

We want to consider non-linear sigma models on homogeneous superspaces $G / G^{\prime}$, where the quotient is defined as the set of right cosets of $G^{\prime}$ in $G$ through the identification

$$
g \sim g h \text { for all } h \in G^{\prime} \subset G \text {. }
$$

Let $\mathfrak{g}$ be the Lie superalgebra associated to $G$. We assume that $\mathfrak{g}$ comes equipped with a non-degenerate invariant bilinear form ( , ). Examples include $\mathfrak{g}=\operatorname{gl}(m \mid n), \operatorname{sl}(m \mid n),^{3}$ $\operatorname{psl}(n \mid n)$ or osp $(m \mid 2 n)$. Similarly, let $\mathfrak{g}^{\prime}$ be the Lie superalgebra associated to $G^{\prime}$. We assume that the restriction of $(,$,$) to \mathfrak{g}^{\prime}$ is non-degenerate. In this case, the orthogonal complement $\mathfrak{m}$ of $\mathfrak{g}^{\prime}$ in $\mathfrak{g}$ is a $\mathfrak{g}^{\prime}$-module and one can write the following $\mathfrak{g}^{\prime}$-module decomposition $\mathfrak{g}=\mathfrak{g}^{\prime} \oplus \mathfrak{m}$. In particular, this means that there are projectors $P^{\prime}$ onto $\mathfrak{g}^{\prime}$ and $P=\mathbb{1}-P^{\prime}$ onto $\mathfrak{m}$ which commute with the action of $\mathfrak{g}^{\prime}$.

With the above requirements, the quotient $G / G^{\prime}$ can be endowed with a $G$-invariant metric $\mathrm{g}$. This metric is by no means unique and generally depends on some number of continuous parameters which we shall also call radii. The square root of the superdeterminant of $\mathrm{g}$ provides in the standard way a $G$-invariant measure $\mu$ on $G / G^{\prime}$. This measure is unique up to a multiplicative constant which depends on the radii of the metric $g$. With these two structures one can already write down a purely kinetic Lagrangian for the sigma model on $G / G^{\prime}$ and quantize it in the path integral formalism. Inclusion of $\theta$-terms, WZW terms or $B$-fields requires a better understanding of the geometry of the $G / G^{\prime}$ superspace. In fact, the $\theta$ and WZW terms are associated to $G$-invariant closed but not exact 2 - and 3 -forms, respectively. $B$-fields, on the other hand, are written in terms of arbitrary $G$ invariant 2-forms that need not be closed. Every such linearly independent form comes with its own coupling constant. We shall only consider Lagrangians with a kinetic term and a $B$-field. Let b be some general linear combination of $G$-invariant exact 2-forms. Then the most general Lagrangian we consider can be written in the form

$$
\mathcal{L}=\eta^{\mu \nu} \mathrm{g}\left(\partial_{\mu}, \partial_{\nu}\right)+\epsilon^{\mu \nu} \mathrm{b}\left(\partial_{\mu}, \partial_{\nu}\right)
$$

where $\eta^{\mu \nu}$ is the constant world sheet metric, $\epsilon^{\mu \nu}$ the antisymmetric tensor with $\epsilon^{01}=1$. The Lagrangian is obviously evaluated on maps from the worldsheet $\Sigma$ to the superspace $G / G^{\prime}$ and to every one of such maps one can associate a vector field $\partial_{\mu}$ on $G / G^{\prime}$, which appears in eq. (2.2) in a coordinate free notation.

There is a different way to formulate the sigma model on $G / G^{\prime}$, which makes its coset nature manifest and allows to explicitly construct the metric $\mathrm{g}$ and the $B$-field $\mathrm{b}$ in eq. (2.2). For that purpose, instead of maps from the worldsheet to the target space $G / G^{\prime}$, we consider more general maps $g: \Sigma \rightarrow G$ from the world sheet to the Lie supergroup $G$. A basis set of 1-forms on $G$ which are invariant under the global left $G$-action is provided by the so called Maurer-Cartan forms

$$
J_{\mu}(x)=g^{-1}(x) \partial_{\mu} g(x) .
$$

\footnotetext{
${ }^{3}$ We exclude $\operatorname{sl}(n \mid n)$ and $\operatorname{pgl}(n \mid n)$, since it does not have a non-degenerate metric.
} 
Higher $G$-invariant tensors may be built out of the Maurer-Cartan forms by taking tensor products. There is a subspace of such tensors which are also invariant with respect to the local right $G^{\prime}$-action. These may be specified by their values on the coset superspace $G / G^{\prime}$. We use this idea in order to build explicitly the $G$-invariant tensors $g$ and b that enter the Lagrangian (2.2).

Under right $G^{\prime}$-gauge transformations $g^{\prime}: \Sigma \mapsto G^{\prime}$ the Maurer-Cartan forms $J_{\mu}$ transform as

$$
g(x) \mapsto g(x) g^{\prime}(x) \quad J_{\mu}(x) \mapsto\left(g^{\prime}(x)\right)^{-1} J_{\mu}(x) g^{\prime}(x)+\left(g^{\prime}(x)\right)^{-1} \partial_{\mu} g^{\prime}(x) .
$$

Since the projection $P$ on $\mathfrak{m}$ commutes with the action of $\mathfrak{g}^{\prime}$, the projected forms $P\left(J_{\mu}\right)$ transforms by conjugation with $g^{\prime}$. To build right $G^{\prime}$-gauge invariant 2 -forms we introduce the $\mathfrak{g}^{\prime}$-intertwiners

$$
G \in \operatorname{End}_{\mathfrak{g}^{\prime}}(\mathfrak{m} \circ \mathfrak{m}, \mathbb{C}) \quad \text { and } \quad B \in \operatorname{End}_{\mathfrak{g}^{\prime}}(\mathfrak{m} \wedge \mathfrak{m}, \mathbb{C})
$$

from the symmetric, respectively antisymmetric tensor product of $\mathfrak{m}$ with itself to the trivial representation. In terms of these intertwiners the Lagrangian (2.2) takes the explicit form

$$
\mathcal{L}=\eta^{\mu \nu} \mathrm{G}\left(P\left(J_{\mu}\right), P\left(J_{\nu}\right)\right)+\epsilon^{\mu \nu} \mathrm{B}\left(P\left(J_{\mu}\right), P\left(J_{\nu}\right)\right) .
$$

The choice of $\mathrm{G}$ and $\mathrm{B}$, subject to some reality constraints, parametrizes the moduli space of the sigma model on $G / G^{\prime}$ with a kinetic term and a $B$-field only. Global left $G$-invariance of the Lagrangian (2.6) is automatic since Maurer-Cartan forms $J_{\mu}(x)$ are left $G$-invariant by construction. Right $G^{\prime}$-gauge invariance, on the other hand, follows easily from the transformation properties of $P\left(J_{\mu}\right)$ and the def. (2.5) of $\mathrm{G}$ and $\mathrm{B}$ as invariant bilinear forms on the $\mathfrak{g}^{\prime}$-module $\mathfrak{m} \otimes \mathfrak{m}$.

\section{$2.2 G / G^{\mathbb{Z}_{N}}$ coset superspaces}

In the previous subsection we have described the most general action with a kinetic term and a $B$-field for the $G$-invariant sigma model with target space $G / G^{\prime}$. The formulation includes sigma models on symmetric spaces and certain generalizations that appear in the context of AdS compactifications. In fact, for many cases of interest, the Lie subsuperalgebra $\mathfrak{g}^{\prime}$ in $\mathfrak{g}$ consists of elements that are invariant under some finite order automorphism $\Omega: \mathfrak{g} \mapsto \mathfrak{g}$. An automorphism of order $N$ defines a decomposition

$$
\mathfrak{g}=\mathfrak{g}^{\prime} \oplus \bigoplus_{i=1}^{N-1} \mathfrak{m}_{i},\left.\quad \Omega\right|_{\mathfrak{g}^{\prime}}=\mathbb{1}, \quad \Omega\left(\mathfrak{m}_{k}\right)=e^{\frac{2 \pi i k}{N}} \mathfrak{m}_{k}
$$

of the superalgebra $\mathfrak{g}$ into eigenspaces of $\Omega$. Extending our previous notation, we denote by $P_{i}$ the projection maps onto $\mathfrak{m}_{i}$. Thanks to the properties of the $\Omega$, we find

$$
\left[\mathfrak{m}_{i}, \mathfrak{m}_{j}\right] \subset \mathfrak{m}_{i+j \bmod N} \quad\left(\mathfrak{m}_{i}, \mathfrak{m}_{j}\right)=0 \quad \text { if } \quad i+j \neq 0 \bmod N
$$

where we have set $\mathfrak{m}_{0} \equiv \mathfrak{g}^{\prime}$. Consequently, the subalgebra $\mathfrak{g}^{\prime}$ acts on the $\Omega$-eigenspaces $\mathfrak{m}_{i}$. Note that the spaces $\mathfrak{m}_{i}$ need not be indecomposable under $\mathfrak{g}^{\prime}$ in which case the decomposition into $\mathfrak{g}^{\prime}$-modules is finer than the decomposition (2.7) into eigenspaces of $\Omega$. 
Whenever a coset superspaces $G / G^{\prime}$ is defined by an automorphism $\Omega$ of order $N$ we shall use the alternative notation $G / G^{\mathbb{Z}_{N}}$. The cases when the grading induced by $\Omega$ is compatible with the $\mathbb{Z}_{2}$ superalgebra grading, that is $\mathfrak{m}_{2 i} \in \mathfrak{g}_{\overline{0}}$ and $\mathfrak{m}_{2 i-1} \in \mathfrak{g}_{\overline{1}}$, were considered by Kagan and Young in [24]. They restricted to a family of Lagrangians for which $\mathrm{G}$ and $\mathrm{B}$ take the following special form

$$
\mathrm{G}(X, Y)=\sum_{i=1}^{N-1} p_{i}\left(P_{i}(X), P_{N-i}(Y)\right), \quad \mathrm{B}(X, Y)=\sum_{i=1}^{N-1} q_{i}\left(P_{i}(X), P_{N-i}(Y)\right),
$$

where the $p_{i}$ and $q_{i}$ are constants obeying the additional constraints

$$
p_{i}=p_{N-i} \quad q_{i}=-q_{N-i} .
$$

The forms of $\mathrm{G}$ and $\mathrm{B}$ in eq. (2.9) do not give rise to the most general Lagrangian for coset superspaces $G / G^{\prime}$. As an example consider the famous $\mathbb{Z}_{4}$ quotient $\operatorname{PSU}(2,2 \mid 4) / \operatorname{SO}(1,4) \times$ $\mathrm{SO}(5)$. Its metric has two radii because its bosonic base is $A d S_{5} \times S^{5}$. On the other hand, the special form of $\mathrm{G}$ in eq. (2.9) allows for only two parameters $p_{1}=p_{3}$ and $p_{2}$, among which $p_{1}$ is redundant because of the purely fermionic nature of $\mathfrak{m}_{1}$ and $\mathfrak{m}_{3}$. In this example, the form that $\mathrm{G}$ takes in eq. (2.9) restricts the radii of $A d S_{5}$ and $S^{5}$ to be equal.

The properties of the theory defined by eqs. (2.9) certainly depend on the precise choice of the parameters $p_{i}$ and $q_{i}$. In particular, it was shown in [25] and [24] that one loop conformal invariance requires

$$
p_{i}=1 \quad q_{i}=1-\frac{2 i}{N} \quad \text { for } i \neq 0,
$$

for all even $N$. We believe, however, that in most cases these conditions are not sufficient to guarantee the vanishing of the full beta function.

Our second comment concerns the treatment of coset superspaces $G / G^{\prime}$ in which the denominator group $G^{\prime}$ has a non-trivial centralizer $Z \subset G$. For such coset superspaces, there exists a residual symmetry by right multiplications with elements of $Z$. In an equivalent formulation one can make all symmetries of $G / G^{\prime}$ to act from the left. For that we rewrite $G / G^{\prime}=G \times Z / G^{\prime} \times Z$ where the factor $Z$ in the denominator is embedded diagonally into the numerator. To make the associated reformulation of the sigma model a bit more explicit, we focus on the principal chiral model for the supergroup $U$. Without any further thought one might be tempted to describe this model through $G=U$ and $G^{\prime}=\{e\}$. But as our introductory comments suggest, we prefer to rewrite the group manifold $U$ as a coset superspace $U=U \times U / U$ and hence to set

$$
G=\{(x, y): x, y \in U\}, \quad G^{\prime}=\{(x, x): x \in U\} .
$$

The left and right action of $G$ on itself is given by componentwise multiplication. The right coset superspace $G / G^{\prime} \cong U$ is considered as the space of equivalence classes under the equivalence relation $(x, y) \sim(x z, y z)$, for all $z \in U$. In particular, $\left(x y^{-1}, 1\right)$ is the canonical representative of the equivalence class of $(x, y)$. Hence, the currents $J_{\mu}$ and the projection map $P: \mathfrak{g} \rightarrow \mathfrak{m}$ are given by

$$
J_{\mu}=\left(x^{-1} \partial_{\mu} x, y^{-1} \partial_{\mu} y\right), \quad P:(v, w) \mapsto\left(\frac{v-w}{2},-\frac{v-w}{2}\right) .
$$


If $(, \quad)$ is the invariant form on the Lie superalgebra of $U$ and we take $\mathrm{G}$ to be given by

$$
\mathbf{G}\left(\left(v_{1}, w_{1}\right) \circ\left(v_{2}, w_{2}\right)\right)=\left(v_{1}, v_{2}\right)+\left(w_{1}, w_{2}\right)
$$

we obtain the usual principal chiral model for $U$. In fact, one may easily show that

$$
\mathrm{G}\left(P\left(J_{\mu}\right), P\left(J_{\nu}\right)\right) \eta^{\mu \nu}=\frac{1}{2}\left(u^{-1} \partial_{\mu} u, u^{-1} \partial_{\nu} u\right) \eta^{\mu \nu},
$$

where $u=x y^{-1} \in U$. Thereby we have established the standard geometric results that allows us to treat the principal chiral model on $U$ as a $G / G^{\prime}$ coset superspace model. The advantage of the seemingly more complicated coset description will become apparent below.

\subsection{Observables and correlators}

We give a brief description of observables and their correlation functions. Let us denote by $\mathcal{G}$ and $\mathcal{G}^{\prime}$ the space of all continuous maps from the world-sheet $\Sigma$ to the supergroups $G$ and $G^{\prime}$, respectively. Obviously, $\mathcal{G}^{\prime}$ acts on $\mathcal{G}$ by point-wise (on $\Sigma$ ) right multiplication. Local observables of the $G / G^{\prime}$ quotient model are defined as some well behaved class of maps $\mathcal{O}: \mathcal{G} \times \Sigma \mapsto \mathbb{C}$ invariant by this right $\mathcal{G}^{\prime}$ action

$$
\mathcal{F}_{G / G^{\prime}}=\left\{\mathcal{O}: \mathcal{G} \times \Sigma \mapsto \mathbb{C} \mid \mathcal{O}(g, x)=\mathcal{O}\left(g \cdot g^{\prime}, x\right) \text { for all } g^{\prime} \in \mathcal{G}^{\prime}\right\}
$$

where we have denoted $\mathcal{O}(g, x):=\mathcal{O}(g(x))$.

One class of observables is obtained by restricting smooth right $G^{\prime}$-invariant functions $f: G \mapsto \mathbb{C}$ to the image of an arbitrary map $g: \Sigma \mapsto G$. Existence of the 2-point function for this observable $f(g(x))$ requires that $f \in L_{2}\left(G / G^{\prime}\right)$. These are the tachyonic fields.

Similarly, all other observables can be obtained from smooth right $G^{\prime}$-invariant tensor forms $t$ of rank $k$ on $G$ by restricting them to the image of some arbitrary map $g: \Sigma \mapsto$ $G$ and evaluating them on the set of vector fields $\partial_{\mu_{1}}, \ldots, \partial_{\mu_{k}}$. Existence of correlation functions for the observables $t_{g(x)}\left(\partial_{\mu_{1}}, \ldots, \partial_{\mu_{k}}\right)$ imposes some further constraints. As an example, let us consider the Maurer-Cartan forms $J_{\mu}$ we have introduced in eq. (2.3). Their components do not give rise to observables of the quotient model because there are not right $G^{\prime}$-gauge invariant. Nevertheless, recalling their behavior (2.4) under right $G^{\prime}$-gauge transformations, one can build the following observables

$$
j_{\mu}=g P\left(J_{\mu}\right) g^{-1} \in \mathcal{F}_{G / G^{\prime}} .
$$

These are the Noether currents for the global symmetry $G$ of the $G / G^{\prime}$ sigma model.

In the following we shall denote by $\mathcal{O}(x)$ the restriction of the local observable $\mathcal{O}$ to the point $x$ of the world-sheet. Given any set $\mathcal{O}_{i} \in \mathcal{F}_{G / G^{\prime}}$ of such local observables we define their unnormalized correlation functions through

$$
\left\langle\prod_{i=1}^{N} \mathcal{O}_{i}\left(x_{i}\right)\right\rangle_{G / G^{\prime}}=\int_{\mathcal{G}}\left[\mathrm{d} \mu_{G}\right] e^{-\mathcal{S}} \prod_{i=1}^{N} \mathcal{O}_{i}\left(x_{i}\right) .
$$


Here, $\mathcal{S}=\int_{\Sigma} d^{2} x \mathcal{L}$ is the action (2.6) of our model. Our definition of correlation functions involves an integration over elements of $\mathcal{G}$ with some left $\mathcal{G}$-invariant measure

$$
\left[d \mu_{G}(g)\right]=\prod_{x \in \Sigma} d \mu_{G}(g(x))
$$

where $d \mu_{G}$ is the unique (up to normalization) Haar measure on $G$. In eq. (2.16), the integration over $G$ at every point of the worldsheet yields a factor which is the volume of $G^{\prime}$. Strictly speaking, this makes sense only if $G^{\prime}$ is compact. We assume that the contribution of such factors can be properly regularized and renormalized by replacing the worldsheet $\Sigma$ with a lattice, and shall not dwell on such details.

The reader might be curious about why we insist on integrating over maps $\mathcal{G}$ from the worldsheet to the group $G$ rather then maps from the worldsheet to the quotient $G / G^{\prime}$. In other words, why we do not fix the right $G^{\prime}$-gauge invariance? As we shall see, keeping this symmetry explicit in the quantum theory simplifies the cohomology calculations on tensor fields.

\section{Cohomological reduction in representation theory}

The following section contains most of the mathematical results we shall need below. Since several of our statements seem to be new, we decided to present and prove them in a rather mathematical style. For pedagogical reasons, however, we shall begin with a short overview of the most relevant notations and results. This should enable impatient readers to skip over subsections 3.2-3.6, at least upon first reading.

\subsection{Overview over results}

As in the previous subsection we assume $\mathfrak{g}$ to be a Lie superalgebra with a non-degenerate symmetric bilinear form ( , ). Let us pick some fermionic element $Q \in \mathfrak{g}$ that squares to zero, i.e. $[Q, Q]=2 Q^{2}=0$. Such elements exist for most Lie superalgebras of interest, with the exception of the series osp $(1 \mid 2 \mathrm{~N})$. The element $Q$ defines a decomposition of $\mathfrak{g}$ into three Lie sub-superalgebras $\mathfrak{h}, \mathfrak{e}, \mathfrak{f}$,

$$
\begin{aligned}
& \mathfrak{g}=\mathfrak{h} \oplus \mathfrak{e} \oplus \mathfrak{f} \quad \text { such that } \\
& \mathfrak{e}=\operatorname{Im}_{Q} \mathfrak{g} \quad \text { and } \quad \mathfrak{h} \oplus \mathfrak{e}=\operatorname{Ker}_{Q} \mathfrak{g} .
\end{aligned}
$$

The bilinear form ( , ) restricts to a non-degenerate form on $\mathfrak{h} \subset \mathfrak{g}$. The Lie subsuperalgebras $\mathfrak{e}$ and $\mathfrak{f}$, on the other hand, are isotropic, i.e. $(\mathfrak{e}, \mathfrak{e})=0=(\mathfrak{f}, \mathfrak{f})$. We also note that $\mathfrak{e}$ and $\mathfrak{f}$ both carry an action of the Lie superalgebra $\mathfrak{h}$.

In subsection 3.2 we shall compute the Lie superalgebra $\mathfrak{h}$ for various choices of $\mathfrak{g}$ and any $Q \in \mathfrak{g}$. The results may be summarized as follows

$$
\begin{aligned}
\mathfrak{h}(\operatorname{gl}(M \mid N)) & \simeq \operatorname{gl}\left(M-r_{Q} \mid N-r_{Q}\right), \\
\mathfrak{h}(\operatorname{sl}(M \mid N)) & \simeq \operatorname{sl}\left(M-r_{Q} \mid N-r_{Q}\right), \\
\mathfrak{h}(\operatorname{osp}(R \mid 2 N)) & \simeq \operatorname{osp}\left(R-2 r_{Q} \mid 2 N-2 r_{Q}\right) .
\end{aligned}
$$


The answer depends on $Q$ only through an integer $\operatorname{rank}(Q)=r_{Q} \geq 1$ that will be defined in section 3.2. In all three cases we listed above, there exist elements $Q$ with minimal rank $r_{Q}=1$.

The element $Q$ acts in any representation $V$ of $\mathfrak{g}$ and defines the following cohomology classes

$$
\mathrm{H}_{Q}(V)=\operatorname{Ker}_{Q} V / \operatorname{Im}_{Q} V
$$

The linear space $\mathrm{H}_{Q}(V)$ comes equipped with an action of the Lie sub-superalgebra $\mathfrak{h} \subset \mathfrak{g}$. It is not difficult to see (cp. section 3.3) that $V \rightarrow \mathrm{H}_{Q}(V)$ is functorial, i.e. it is consistent with forming tensor products, direct sums and conjugation in the category of $\mathfrak{h}$-representations.

Though $\mathrm{H}_{Q}(V)$ vanishes for a large class of representations (see below), it can certainly contain non-trivial elements. Note, for example, that the cohomology of the adjoint $\mathfrak{g}$ module $V=\mathfrak{g}$ is given by $\mathrm{H}_{Q}(\mathfrak{g})=\mathfrak{h}$. One may actually show that $V$ and $\mathrm{H}_{Q}(V)$ possess the same super-dimension. Hence, all representations $V$ with non-vanishing super-dimension $\operatorname{sdim} V=\operatorname{dim} V_{\overline{0}}-\operatorname{dim} V_{\overline{1}}$ must give rise to $\mathrm{H}_{Q}(V) \neq 0$. The condition $\operatorname{sdim} V \neq 0$ is often satisfied for short multiplets (atypical representations). For long (typical) multiplets $V$, on the other hand, the cohomology $\mathrm{H}_{Q}(V)$ is always trivial. More generally, we will see that $\mathrm{H}_{Q}(V)=0$ for all (finite dimensional) projective modules.

Let us now consider a Lie superalgebra $\mathfrak{g}$ along with a subalgebra $\mathfrak{g}^{\prime} \subset \mathfrak{g}$. The corresponding Lie supergroups will be denoted by $G$ and $G^{\prime}$, respectively. As before, we want to pick some fermionic element $Q \in \mathfrak{g}$ with $Q^{2}=0$. Let us now assume that $Q$ is contained in the subalgebra $\mathfrak{g}^{\prime} \subset \mathfrak{g}$ so that its cohomology defines two Lie sub-superalgebras $\mathfrak{h} \subset \mathfrak{g}$ and $\mathfrak{h}^{\prime} \subset \mathfrak{g}^{\prime}$ with $\mathfrak{h}^{\prime} \subset \mathfrak{h}$. We denote the associated Lie supergroups by $H$ and $H^{\prime}$, respectively. Note that the space of functions on the coset superspace $G / G^{\prime}$ carries an action of $\mathfrak{g}$. In particular, the element $Q$ acts and gives rise to some cohomology. The central claim of this section is that the cohomology of some geometric object (smooth function, tensor form, square integrable function) defined on the coset superspace $G / G^{\prime}$ is equivalent to a similar object defined on $H / H^{\prime}$. This gives rise to isomorphisms of the type

$$
\mathrm{H}_{Q}\left(L_{2}\left(G / G^{\prime}\right)\right) \cong L_{2}\left(H / H^{\prime}\right)
$$

which means that the cohomology of $Q$ in the space of square integrable functions on $G / G^{\prime}$ may be interpreted as a space of square integrable functions on the coset superspace $H / H^{\prime}$. We note that $L_{2}\left(H / H^{\prime}\right)$ carries an action of the Lie superalgebra $\mathfrak{h}=\mathrm{H}_{Q}(\mathfrak{g}) \subset \mathfrak{g}$. The isomorphism (3.4) is an isomorphism of $\mathfrak{h}$ modules.

The derivation of eq. (3.4) is a bit involved. We shall provide a fully explicit proof in section 3.6. Here, we shall content ourselves with some more qualitative arguments. By construction, $\mathrm{H}_{Q}\left(L_{2}\left(G / G^{\prime}\right)\right)$ is a commutative algebra and hence it can be considered as an algebra of functions on some space $X$. The latter is acted upon by the supergroup $H$ with Lie superalgebra $\mathrm{H}_{Q}(\mathfrak{g})=\mathfrak{h}$. Since the action of $G$ on $G / G^{\prime}$ is transitive, it suffices to understand the reduction from $G / G^{\prime}$ to $X$ locally, near the image $e G^{\prime} \in G / G^{\prime}$ of the group unit $e \in G$. The tangent space at this point of the coset supermanifold is given by $\mathfrak{g} / \mathfrak{g}^{\prime} \equiv \mathfrak{m}$. Its cohomology is given by

$$
\mathrm{H}_{Q}(\mathfrak{m})=\mathrm{H}_{Q}\left(\mathfrak{g} / \mathfrak{g}^{\prime}\right)=\mathrm{H}_{Q}(\mathfrak{g}) / \mathrm{H}_{Q}\left(\mathfrak{g}^{\prime}\right)=\mathfrak{h} / \mathfrak{h}^{\prime},
$$


i.e. the tangent vectors to the reduced space $X$ lie in $\mathfrak{h} / \mathfrak{h}^{\prime}$. Thereby we conclude that $X=H / H^{\prime}$. Now, let $\langle,\rangle_{G / G^{\prime}}$ be the $G$-invariant scalar product of $L_{2}\left(G / G^{\prime}\right)$. It is very easy to see that $\langle,\rangle_{G / G^{\prime}}$ descends to cohomology. Hence, the space $\mathrm{H}_{Q}\left(L_{2}\left(G / G^{\prime}\right)\right)$ of functions inherits an $L_{2}$ structure from $L_{2}\left(G / G^{\prime}\right)$. We shall denote it by $\langle,\rangle_{H / H^{\prime}}$. Its $H$-invariance follows immediately from the $G$-invariance of $\langle,\rangle_{G / G^{\prime}}$ and the inclusion $\mathfrak{h} \subset \operatorname{Ker}_{Q} \mathfrak{g}$. General results on measure theory [26], ${ }^{4}$ then imply that the scalar product $\langle,\rangle_{H / H^{\prime}}$ arises from a measure on $H / H^{\prime}$, which is unique (up to a constant factor) by $H$-invariance. Hence, we have established eq. (3.4).

As an example of the above, let us discuss the Lie superalgebra $\mathfrak{g}=\operatorname{gl}(2 \mid 2)$. For $Q$ we pick the supermatrix that contains a single entry in the upper right corner. It is then easy to check that

$$
\operatorname{Ker}_{Q} \mathfrak{g}=\mathfrak{h} \oplus \mathfrak{e} \ni\left(\begin{array}{cc|cc}
a_{11} & a_{12} & b_{11} & b_{12} \\
0 & a_{22} & b_{21} & b_{22} \\
\hline 0 & c_{12} & d_{11} & d_{12} \\
0 & 0 & 0 & a_{11}
\end{array}\right), \quad \operatorname{Im}_{Q} \mathfrak{g}=\mathfrak{e} \ni\left(\begin{array}{cc|cc}
a_{11} & a_{12} & b_{11} & b_{12} \\
0 & 0 & 0 & b_{22} \\
\hline 0 & 0 & 0 & d_{12} \\
0 & 0 & 0 & a_{11}
\end{array}\right)
$$

Consequently, $\mathrm{H}_{Q}(\mathfrak{g})=\mathfrak{h}=\operatorname{gl}(1 \mid 1)$ consists of all supermatrices in which $a_{22}, b_{21}, c_{12}$ and $d_{11}$ are the only non-vanishing entries. Let us also specify the Lie sub-superalgebra $\mathfrak{g}^{\prime}$ to consist of all elements in $\mathfrak{g}$ with vanishing entries $b_{11}=b_{21}=d_{12}=d_{21}=c_{11}=c_{12}=0$. Hence, $\mathfrak{g}^{\prime} \cong \operatorname{gl}(2 \mid 1) \times \operatorname{gl}(1)$. The cohomology $\mathrm{H}_{Q}\left(\mathfrak{g}^{\prime}\right)=\mathfrak{h}^{\prime}=\operatorname{gl}(1) \times \operatorname{gl}(1)$ of $\mathfrak{g}^{\prime}$ can be read off easily.

In our example, the quotient $G / G^{\prime}$ is the complex projective superspace $\mathbb{C P}^{1 / 2} \cong$ $S^{2} \times \mathbb{R}^{0 \mid 4}$. Functions thereon may be decomposed into finite dimensional representations of $\operatorname{psl}(2 \mid 2)$ as follows

$$
L_{2}\left(\mathbb{C P}^{1 \mid 2}\right) \cong \bigoplus_{j=0}^{\infty}[j, 0] .
$$

The representations $[j, 0]$ of $\operatorname{psl}(2 \mid 2)$ that appear in this decomposition possess dimension $d_{j}=16(2 j+1)$. They are generated from the spherical harmonics on the bosonic 2 -sphere by application of four fermionic generators. For $j \neq 0$, the $\operatorname{psl}(2 \mid 2)$ modules $[j, 0]$ turn out to be projective (typical long multiplets) and hence $\mathrm{H}_{Q}([j, 0])=0$ for all $j \neq 0$. The only non-vanishing cohomology comes from the 16-dimensional Kac module [0,0]. The latter is built from three atypical irreducibles, namely two copies of the trivial representation and one copy of the 14-dimensional adjoint representation of $\operatorname{psl}(2 \mid 2)$. Each of these pieces contributes to cohomology. While the two trivial representations give rise to two even states, the adjoint representation has an excess of two odd states which descend to cohomology. In total, we obtain a 4-dimensional cohomology

$$
\mathrm{H}_{Q}\left(L_{2}\left(G / G^{\prime}\right)\right)=\mathrm{H}_{Q}\left(L_{2}\left(\mathbb{C P}^{1 \mid 2}\right)\right)=\mathrm{H}_{Q}([0,0])=\mathbb{R}^{2 \mid 2}
$$

To me more precise, we note that the linear space $\mathbb{R}^{2 \mid 2}$ carries the 4-dimensional projective cover of gl(1|1). According to our general statement, the cohomology should agree

\footnotetext{
${ }^{4}$ For non-compact spaces we implicitely assumed we are dealing with functions vanishing at infinity. See the Riesz-Markov theorem on page 111 of [26].
} 
with the space of functions on the quotient $H / H^{\prime}=\mathrm{GL}(1 \mid 1) / \mathrm{GL}(1) \times \mathrm{GL}(1)$. The quotient possesses two fermionic coordinates and hence gives rise to a 4-dimensional algebra of functions over it,

$$
L_{2}\left(H / H^{\prime}\right)=\mathbb{R}^{2 \mid 2} .
$$

It indeed agrees with the cohomology in the space of functions over $\mathbb{C P}^{1 \mid 2}$, as it was claimed in eq. (3.4).

\subsection{Reduction of Lie superalgebras}

As in the previous subsection, let $\mathfrak{g}$ denote a Lie superalgebra and $Q$ be any fermionic element of $\mathfrak{g}$ with vanishing bracket, that is $[Q, Q]=2 Q^{2}=0$.

Lemma 1. The element $Q \in \mathfrak{g}$ gives rise to a linear map $Q: \mathfrak{g} \rightarrow \mathfrak{g}$ that is defined by $Q(X)=[Q, X]$ for all $X \in \mathfrak{g}$. Then it is possible to show that

1) the subspaces $\operatorname{Ker}_{Q} \mathfrak{g}$ and $\operatorname{Im}_{Q} \mathfrak{g}$ are subalgebras of $\mathfrak{g}$,

2) the subalgebra $\operatorname{Im}_{Q} \mathfrak{g}$ is an ideal of $\operatorname{Ker}_{Q} \mathfrak{g}$,

3) the quotient space $H_{Q}(\mathfrak{g})$ is a Lie superalgebra.

All assertions of this lemma are easily established using no more that the (graded) Jacobi identity. The Lie bracket on the quotient space $\mathrm{H}_{Q}(\mathfrak{g})$ is induced from the Lie bracket of $\mathfrak{g}$ through

$$
\left[x+\operatorname{Im}_{Q} \mathfrak{g}, y+\operatorname{Im}_{Q} \mathfrak{g}\right]=[x, y]+\operatorname{Im}_{Q} \mathfrak{g}, \quad x, y \in \operatorname{Ker}_{Q} \mathfrak{g} .
$$

We shall often refer to the space $\mathrm{H}_{Q}(\mathfrak{g})$ as the cohomological reduction of the Lie superalgebra $\mathfrak{g}$ with respect to $Q$. In our discussion of concrete examples we shall essentially restrict to the superalgebras $\mathfrak{g}$ of the type osp $(M \mid 2 N), \operatorname{gl}(M \mid N)$ or $\operatorname{sl}(M \mid N), N \neq M$. All these Lie superalgebras possess an invariant, consistent, supersymmetric, non-degenerate bilinear form $(, \quad): \mathfrak{g} \times \mathfrak{g} \rightarrow \mathbb{C}$.

The adjoint action of $Q$ can be brought in its Jordan normal form by choosing a basis $\left\{h_{a}\right\} \cup\left\{e_{i}, f_{i}\right\}$ of $\mathfrak{g}$ such that

$$
\left[Q, h_{a}\right]=0 \quad \text { and } \quad\left[Q, f_{i}\right]=e_{i}
$$

Using the invariance of the bilinear form we show that

$$
\left(h_{a}, e_{i}\right)=0, \quad\left(e_{i}, e_{j}\right)=0 .
$$

If follows from the non-degeneracy of the bilinear form that the matrix $D_{i j}=\left(e_{i}, f_{j}\right)$ must be invertible. Defining

$$
\begin{aligned}
h_{a}^{\prime} & =h_{a}-\left(h_{a}, f_{j}\right)\left(D^{-1}\right)^{j i} e_{i}, \\
f_{i}^{\prime} & =f_{i}-\frac{1}{2}\left(f_{i}, f_{j}\right)\left(D^{-1}\right)^{j k} e_{k}
\end{aligned}
$$


we see that

$$
\left(h_{a}^{\prime}, f_{i}^{\prime}\right)=0, \quad\left(f_{i}^{\prime}, f_{j}^{\prime}\right)=0 .
$$

To prove the second assertion in eq. (3.11) we have used the following property of the matrix $D$

$$
D_{i j}=\left(\left[Q, f_{i}\right], f_{j}\right)=-(-1)^{\left|f_{i}\right|}\left(f_{i},\left[Q, f_{j}\right]\right)=-(-1)^{\left|f_{i}\right|}\left(f_{i}, e_{j}\right)=-D_{j i},
$$

where the last equality in the chain uses the consistency of the bilinear form.

Let us denote by $\mathfrak{h}, \mathfrak{e}$ and $\mathfrak{f}$ the span of $h_{a}^{\prime}, e_{i}$ and $f_{i}^{\prime}$, respectively. Notice that $Q$ still remains in a Jordan normal form with respect to the new basis $h_{a}^{\prime}, e_{i}, f_{j}^{\prime}$. From the eqs. (3.8), (3.11) we deduce the following orthogonality conditions

$$
(\mathfrak{h}, \mathfrak{e})=(\mathfrak{h}, \mathfrak{f})=(\mathfrak{e}, \mathfrak{e})=(\mathfrak{f}, \mathfrak{f})=0 .
$$

Using once more the invariance of the bilinear form it is not hard to derive the following features of the Lie bracket on $\mathfrak{g}$,

$$
\begin{aligned}
& {[\mathfrak{h}, \mathfrak{h}] \subset \mathfrak{h}, \quad[\mathfrak{h}, \mathfrak{e}] \subset \mathfrak{e}, \quad[\mathfrak{h}, \mathfrak{f}] \subset \mathfrak{f},} \\
& {[\mathfrak{e}, \mathfrak{e}] \subset \mathfrak{e}, \quad[\mathfrak{f}, \mathfrak{f}] \subset \mathfrak{f}, \quad[\mathfrak{e}, \mathfrak{f}] \subset \mathfrak{g} .}
\end{aligned}
$$

Notice, in particular, that both $\mathfrak{e}$ and $\mathfrak{f}$ provide some representation for the Lie superalgebra $\mathfrak{h}$. Furthermore, we observe that $\mathfrak{g}$ and $\mathfrak{h}$ possess the same cohomology, $\mathrm{H}_{Q}(\mathfrak{g})=\mathrm{H}_{Q}(\mathfrak{h})$. Next, let us define the projection map $p_{\mathfrak{h}}: \mathfrak{g} \rightarrow \mathfrak{h}$ through

$$
p_{\mathfrak{h}}(x)=\left(x, h_{a}^{\prime}\right)\left(B^{-1}\right)^{a b} h_{b}^{\prime},
$$

where $x \in \operatorname{Ker}_{Q}$ and $B_{a b}=\left(h_{a}^{\prime}, h_{b}^{\prime}\right)$. The kernel of $p_{\mathfrak{h}}$ being exactly $\mathfrak{e}$, the map $p_{\mathfrak{h}}$ is effectively defined on $\mathrm{H}_{Q}(\mathfrak{g})$. Taking into account eqs. (3.14), we see that $p_{\mathfrak{h}}$ provides the following algebra isomorphism

$$
\mathfrak{h} \simeq \mathrm{H}_{Q}(\mathfrak{g}) .
$$

In the same spirit, one can define $\mathfrak{h}$-module projection homomorphisms $p_{\mathfrak{e}}$ and $p_{\mathfrak{f}}$ from $\mathfrak{g}$ to $\mathfrak{e}$ and $\mathfrak{f}$, respectively,

$$
\begin{aligned}
& p_{\mathfrak{e}}(x)=\left(x, f_{i}^{\prime}\right)\left(D^{-1}\right)^{i j} e_{j} \\
& p_{\mathfrak{f}}(x)=x-p_{\mathfrak{h}}(x)-p_{\mathfrak{e}}(x) .
\end{aligned}
$$

These provide the following direct sum decomposition of $\mathfrak{g}$,

$$
\mathfrak{g} \simeq \mathfrak{h} \oplus \mathfrak{e} \oplus \mathfrak{f} .
$$

The isomorphism respects the action of $\mathfrak{h}$, i.e. it is an isomorphism of $\mathfrak{h}$ modules.

The superalgebras we consider are characterized by a Cartan subalgebra which we denote, in a somewhat non-standard way, by $\mathfrak{g}_{0}$ and a root system $\Delta$. If $R: \mathfrak{g} \rightarrow \mathrm{gl}(\mathrm{V})$ is the fundamental representation, then the Cartan subalgebra $\mathfrak{g}_{0}$ can be represented through diagonal matrices of $\mathrm{gl}(\mathrm{V})$, while $\Delta$ is a subset of the root system of $\mathrm{gl}(\mathrm{V})$. 


\begin{tabular}{|lcc|}
\hline $\mathfrak{g}$ & $\Delta_{\overline{0}}$ & $\Delta_{\overline{1}}$ \\
\hline $\operatorname{gl}(M \mid N), \operatorname{sl}(M \mid N)$ & $\epsilon_{i}-\epsilon_{j}$ & \\
& $\delta_{k}-\delta_{l}$ & $\pm \epsilon_{i} \mp \delta_{k}$ \\
& & \\
& $\pm \epsilon_{i} \pm \epsilon_{j}$ & \\
$\operatorname{osp}(2 M \mid 2 N)$ & $\pm \delta_{k} \pm \delta_{l}$ & $\pm \epsilon_{i} \pm \delta_{k}$ \\
& $\pm 2 \delta_{k}$ & \\
& & \\
& $\pm \epsilon_{i} \pm \epsilon_{j}$ & \\
& $\pm \epsilon_{i}$ & $\pm \epsilon_{i} \pm \delta_{k}$ \\
& $\pm \delta_{k} \pm \delta_{l}$ & $\pm \delta_{k}$ \\
& $\pm 2 \delta_{k}$ & \\
\hline & \multicolumn{2}{c}{} \\
& &
\end{tabular}

Table 1. The root systems of $\mathrm{gl}$, sl and osp type superalgebras in the standard basis $\epsilon_{1}, \ldots, \epsilon_{M}, \delta_{1}, \ldots, \delta_{M}$. See for instance [27] for more details.

Let us now perform the cohomological reduction for the Lie superalgebra $\mathfrak{g}$ when $Q$ is a root generator of root $q$ such that $2 q \notin \Delta$. Consider the root decomposition of $\mathfrak{g}$

$$
\mathfrak{g}=\mathfrak{g}_{0} \oplus \bigoplus_{\alpha \in \Delta} \mathfrak{g}_{\alpha} .
$$

The superalgebras $\mathfrak{e}$ and $\mathfrak{f}$ can be easily evaluated

$$
\begin{aligned}
& \mathfrak{e}=\mathbb{C} H_{q} \oplus \bigoplus_{\alpha-q \in \Delta} \mathfrak{g}_{\alpha}, \\
& \mathfrak{f}=\mathfrak{g}_{0} / \operatorname{Ker} q \oplus \bigoplus_{\alpha+q \in \Delta} \mathfrak{g}_{\alpha},
\end{aligned}
$$

where for any weight $\lambda$ one denotes by $H_{\lambda}$ the Cartan generator constructed through

$$
\lambda(H)=\left(H_{\lambda}, H\right) .
$$

Therefore, we can write the cohomology of $\mathfrak{g}$ in the form

$$
\mathrm{H}_{q}(\mathfrak{g}):=\mathrm{H}_{Q}(\mathfrak{g}) \simeq \mathfrak{h}=\operatorname{Ker} q / \mathbb{C} H_{q} \oplus \bigoplus_{\alpha \pm q \notin \Delta} \mathfrak{g}_{\alpha} .
$$

Let us apply this general result to compute the cohomological reduction of the superalgebras gl, sl and osp. For the readers convenience we have listed the relevant root systems in table 1.

Consider the superalgebra $\operatorname{gl}(M \mid N)$ first. Let $Q$ be a root generator for the root $q=\epsilon_{r}-\delta_{s}$. The requirement $\alpha \pm q \notin \Delta$ is satisfied for the following roots

$$
\epsilon_{i}-\epsilon_{j}, \epsilon_{i}-\delta_{k}, \quad \text { with } \quad \delta_{k}-\delta_{l}, \quad i, j \neq r, k, l \neq s .
$$


These give rise to the root system of a $\mathrm{gl}(M-1 \mid N-1)$ subalgebra. As a basis of the Cartan subalgebra one may choose the Cartan generators $H_{\epsilon_{i}}, H_{\delta_{k}}$ which are defined through eq. (3.22). Evaluating

$$
\operatorname{Ker}\left(\epsilon_{r}-\delta_{s}\right) / \mathbb{C} H_{\epsilon_{r}-\delta_{s}}=\operatorname{Ker}\left(\epsilon_{r}-\delta_{s}\right) \cap \operatorname{Ker}\left(\epsilon_{r}+\delta_{s}\right)=\operatorname{Ker} \epsilon_{r} \cap \operatorname{Ker} \delta_{s}
$$

we deduce with the help of eq. (3.23) that

$$
\mathrm{H}_{\epsilon_{r}-\delta_{s}}(\mathrm{gl}(M \mid N)) \simeq \operatorname{gl}(M-1 \mid N-1) .
$$

The cohomological reduction of $\operatorname{sl}(M \mid N)$ is only slightly different. As the roots of $\operatorname{sl}(M \mid N)$ and $\operatorname{gl}(M \mid N)$ are the same, the analysis (3.24) remains unchanged. The Cartan algebra of $\operatorname{sl}(M \mid N)$ can be viewed as the subalgebra of the Cartan algebra of $\operatorname{gl}(M \mid N)$ defined by Ker str, where we have introduced the supertrace str $:=\sum \epsilon_{i}-\sum \delta_{k}$. Therefore, eq. (3.25) has to be replaced by

$$
\begin{aligned}
\operatorname{Kerstr} \cap \operatorname{Ker}\left(\epsilon_{r}-\delta_{s}\right) / \mathbb{C} H_{\epsilon_{r}-\delta_{s}} & =\operatorname{Kerstr} \cap \operatorname{Ker}\left(\epsilon_{r}-\delta_{s}\right) \cap \operatorname{Ker}\left(\epsilon_{r}+\delta_{s}\right) \\
& =\operatorname{Ker}\left(\sum_{i \neq r} \epsilon_{i}-\sum_{k \neq s} \delta_{k}\right) \cap \operatorname{Ker} \epsilon_{r} \cap \operatorname{Ker} \delta_{s},
\end{aligned}
$$

which leads to the Cartan subalgebra of $\operatorname{sl}(M-1 \mid N-1)$. Therefore we obtain

$$
\mathrm{H}_{\epsilon_{r}-\delta_{s}}(\operatorname{sl}(M \mid N)) \simeq \operatorname{sl}(M-1 \mid N-1) .
$$

A similar analysis may be performed for osp type superalgebras. If we choose $q=\epsilon_{r} \pm \delta_{s}$ then $\alpha \pm q$ is not a root for all $\alpha$ from the following list

$$
\pm \epsilon_{i} \pm \epsilon_{j}, \quad \pm \epsilon_{i} \pm \delta_{k}, \quad \pm \delta_{k} \pm \delta_{l}, \quad i \neq j, \quad i, j \neq r, \quad k, l \neq s
$$

in the case of osp $(2 M \mid 2 N)$ and

$$
\pm \epsilon_{i} \pm \epsilon_{j}, \quad \pm \epsilon_{i} \pm \epsilon_{i} \pm \delta_{k}, \quad \pm \delta_{k} \pm \delta_{l}, \quad i \neq j, \quad i, j \neq r, \quad k, l \neq s,
$$

in the case of osp $(2 M+1 \mid 2 N)$. Those in eq. (3.28) correspond to the root system of an osp $(2 M-2 \mid 2 N-2)$ subalgebra, while the roots in eq. (3.29) are associated with an osp $(2 M-1 \mid 2 N-2)$ subalgebra. Again, one may take the Cartan Cartan generators $H_{\epsilon_{i}}$, $H_{\delta_{k}}$ as a basis of the Cartan subalgebra. The cohomological reduction of the Cartan subalgebra goes exactly as in eq. (3.25)

$$
\operatorname{Ker}\left(\epsilon_{r} \pm \epsilon_{r}\right) / \mathbb{C} H_{\epsilon_{r} \pm \delta_{s}}=\operatorname{Ker}\left(\epsilon_{r} \pm \epsilon_{r}\right) \cap \operatorname{Ker}\left(\epsilon_{r} \mp \epsilon_{r}\right)=\operatorname{Ker}_{\epsilon_{r}} \cap \operatorname{Ker}_{\delta_{s}}
$$

Therefore we conclude that

$$
\mathrm{H}_{\epsilon_{r} \pm \delta_{s}}(\operatorname{osp}(R \mid 2 N)) \simeq \operatorname{osp}(R-2 \mid 2 N-2)
$$

for any choice of $R$. At this point we have determined the cohomology $H_{Q}(\mathfrak{g})$ for all elements $Q$ that belong to the Cartan eigenspace $\mathfrak{g}_{q}$ of an isotropic root $q$. 
From eqs. (3.26), (3.27), (3.31) we may infer that, up to isomorphism, the cohomological reduction of $\mathfrak{g}$ with respect to $Q$ does not depend on the choice of the isotropic root $q$. This gives rise to the following question: How can we characterize $Q \mathrm{~s}$ that give rise to different Lie superalgebras $\mathrm{H}_{Q}(\mathfrak{g})$ ? In the following we want to prove that the isomorphism class of the cohomological reduction depends only on the rank of $Q$ in the fundamental representation. To begin with we observe that an automorphism $\gamma$ of $\mathfrak{g}$ induces an automorphism of the cohomology, i.e.

$$
\mathrm{H}_{Q}(\mathfrak{g}) \simeq \mathrm{H}_{\gamma(Q)}(\mathfrak{g})
$$

The main idea is to use the group of inner automorphisms provided by the even subalgebra of $\mathfrak{g}$ in order to bring a general $Q$ with vanishing self-bracket to some simpler form.

Consider the Lie superalgebra $\mathrm{gl}(M \mid N)$ first. Let $V, V_{M}$ and $V_{N}$ be the fundamental $\mathrm{gl}(M \mid N), \mathrm{gl}(\mathrm{M})$ and $\mathrm{gl}(\mathrm{M})$ modules, respectively. To bring $Q$ to some simpler form, we shall use the following $\mathrm{gl}(M \mid N)_{\overline{0}} \simeq \mathrm{gl}(\mathrm{M}) \oplus \mathrm{gl}(\mathrm{N})$ module isomorphism

$$
\operatorname{gl}(M \mid N)_{\overline{1}} \simeq V_{M} \otimes_{\mathbb{C}} V_{N}^{*} \oplus V_{N} \otimes_{\mathbb{C}} V_{M}^{*}
$$

where $V^{*}$ denotes the dual representation. The module isomorphism (3.33) is provided by the invertible linear map

$$
\begin{aligned}
\varphi(v \otimes \alpha)(a) & =v \alpha(a), & v \otimes \alpha & \in V_{M} \otimes \mathbb{C} V_{N}^{*} \\
\varphi(v \otimes \alpha)(u) & =0, & u & \in V_{M} \\
\varphi(a \otimes \omega)(v) & =a \omega(v), & a \otimes \omega & \in V_{N} \otimes_{\mathbb{C}} V_{M}^{*} \\
\varphi(a \otimes \omega)(b) & =0, & b & \in V_{N} .
\end{aligned}
$$

We say that $Q$ has rank $(k, l)$ if it can be represented as

$$
\varphi^{-1}(Q)=\sum_{i=1}^{k} v_{i} \otimes \alpha^{i}+\sum_{i=1}^{l} a_{i} \otimes \omega^{i},
$$

where all $v$ 's, $a$ 's, $\alpha$ 's and $\omega$ 's are linearly independent among themselves. Clearly $k, l \leq$ $\min (M, N)$. Let $b_{1}, \ldots, b_{M}$ denote a basis of $V_{M}$ and $f_{1}, \ldots, f_{N}$ be a basis of $V_{N}$. Denote by $b^{i}, f^{k}$ the dual bases. Then, from the definition of the general linear group, there are elements $A^{\prime} \in \mathrm{GL}(\mathrm{M}), B^{\prime} \in \mathrm{GL}(\mathrm{N})$ such that

$$
v_{i}=A^{\prime} \cdot b_{i}, \quad \alpha^{i}=B^{\prime} \cdot f^{i}, \quad i=1, \ldots, k .
$$

Moreover, the group elements $A^{\prime}, B^{\prime}$ are not unique - their action on the remaining basis vectors $b_{k+1}, \ldots, b_{M}$ and $f^{k+1}, \ldots, f^{N}$ is not fixed. Choosing an inner automorphism $\gamma^{\prime}=$ $\operatorname{Ad} A^{\prime-1} \circ \operatorname{Ad} B^{\prime-1}$ we see that one can bring $Q$ to the simpler form

$$
\varphi^{-1}\left(\gamma^{\prime}(Q)\right)=\sum_{i=1}^{k} b_{i} \otimes f^{i}+\sum_{i=1}^{l} a_{i}^{\prime} \otimes \omega^{i},
$$


where $a_{i}^{\prime}=B^{\prime-1} \cdot a_{i}$ and $\omega^{\prime i}=A^{\prime-1} \cdot \omega^{i}$. The condition $Q^{2}=0$ is equivalent to the following constraints on the vectors $a_{i}^{\prime}, \omega^{\prime i}$ in eq. (3.37)

$$
f^{j}\left(a_{i}^{\prime}\right)=0, \quad \omega^{\prime i}\left(b_{j}\right)=0,
$$

where $i=1, \ldots, l, j=1, \ldots, k$. This means that the vectors $a_{i}^{\prime}$ lie entirely in the subspace of $V_{N}$ spanned by the basis vectors $f_{k+1}, \ldots, f_{N}$, while the form $\omega^{\prime i}$ lies in the subspace of $V_{M}^{*}$ that is spanned by the basis forms $b^{k+1}, \ldots, b^{M}$. Therefore, the linear independence of $a_{i}^{\prime}, \omega^{\prime i}$ imposes an additional restriction on the rank $(k, l)$ of $Q$

$$
k+l \leq \min (M, N) .
$$

The existence of the group elements $A^{\prime \prime} \in \mathrm{GL}(\mathrm{M})$ and $B^{\prime \prime} \in \mathrm{GL}(\mathrm{N})$ satisfying

$$
A^{\prime \prime} \cdot b_{i}=b_{i}, \quad B^{\prime \prime} \cdot f^{i}=f^{i},
$$

for $i=1, \ldots, k$ and

$$
a_{m}^{\prime}=A^{\prime \prime} \cdot f_{m}, \quad \omega^{\prime n}=B^{\prime \prime} \cdot b^{n},
$$

for $m=k+1, \ldots, k+l$ and $n=k+1, \ldots, k+l$ is ensured by eq. (3.38). Defining $\gamma^{\prime \prime}=\operatorname{Ad} A^{\prime \prime-1} \circ \operatorname{Ad} B^{\prime \prime-1}$ we see that $Q$ can be brought into a standard form which depends only on its rank $(k, l)$

$$
\varphi^{-1}\left(\left(\gamma^{\prime \prime} \circ \gamma^{\prime}\right)(Q)\right)=\sum_{i=1}^{k} b_{i} \otimes f^{i}+\sum_{i=k+1}^{k+l} f_{i} \otimes b^{i} .
$$

We perform the cohomological reduction of $\mathrm{gl}(M \mid N)$ with respect to the fermionic generators

$$
\varphi\left(\sum_{i=1}^{k} b_{i} \otimes f^{i}+\sum_{i=k+1}^{k+l} f_{i} \otimes b^{i}\right)
$$

by a lengthy but straightforward calculation. Thereby, we are lead to the following statement

$$
\mathrm{H}_{Q}(\mathrm{gl}(M \mid N)) \simeq \operatorname{gl}(M-\operatorname{rank}(Q) \mid N-\operatorname{rank}(Q)),
$$

where the total rank of $Q$ is defined as $\operatorname{rank}(Q)=k+l \leq \min (M, N)$.

The generalization to the superalgebras $\operatorname{sl}(M \mid N)$ is straightforward. The procedure to bring $Q$ to the canonical form (3.42) is identical with the one described in the $\mathrm{gl}(M \mid N)$ case. The cohomological reduction of $\operatorname{sl}(M \mid N)$ with respect to this canonical form of $Q$ may be performed explicitly and leads to the expected result

$$
\mathrm{H}_{Q}(\operatorname{sl}(M \mid N)) \simeq \operatorname{sl}(M-\operatorname{rank}(Q) \mid N-\operatorname{rank}(Q)) .
$$

Finally, let us also deal with the Lie superalgebras $\operatorname{osp}(R \mid 2 N)$, where $R=2 M$ or $R=2 M+1$. Denote by $V, V_{R}$ and $V_{2 N}$ the fundamental osp $(R \mid 2 N)$, so $(\mathrm{R})$ and $\operatorname{sp}(2 \mathrm{~N})$ modules, respectively. Furthermore, let $($,$) be the symmetric invariant scalar product$ in $V_{R}$ and $\langle$,$\rangle be the antisymmetric invariant scalar product in V_{2 N}$. For $R=2 M$ 
we shall consider a basis $b_{1}, \ldots, b_{2 M}$ such that the matrix elements of the scalar product $S_{i j}=\left(b_{i}, b_{j}\right)$ take the form

$$
S=\left(\begin{array}{ll}
0_{M \times M} & 1_{M \times M} \\
1_{M \times M} & 0_{M \times M}
\end{array}\right),
$$

while for $R=2 M+1$ we shall consider a basis $b_{1}, \ldots, b_{2 M+1}$ such that the matrix elements of the scalar product $S_{i j}=\left(b_{i}, b_{j}\right)$ take the form

$$
S=\left(\begin{array}{ccc}
0_{M \times M} & 1_{M \times M} & 0_{M \times 1} \\
1_{M \times M} & 0_{M \times M} & 0_{M \times 1} \\
0_{1 \times M} & 0_{1 \times M} & 1
\end{array}\right) .
$$

We also consider a basis $f_{1}, \ldots, f_{2 N}$ such that the matrix elements of the scalar product $A_{i j}=\left\langle f_{i}, f_{j}\right\rangle$ take the form

$$
A=\left(\begin{array}{cc}
0_{N \times N} & -1_{N \times N} \\
1_{N \times N} & 0_{N \times N}
\end{array}\right) .
$$

With respect to the decomposition $V \simeq V_{R} \oplus V_{2 N}$, the invariant scalar product in $V$ is $G=S \oplus A$.

To bring $Q$ into some simpler form, we shall use the following osp $(R \mid 2 N)_{\overline{0}} \simeq \operatorname{so}(\mathrm{R}) \oplus$ $\operatorname{sp}(2 \mathrm{~N})$ module isomorphism

$$
\operatorname{osp}(R \mid 2 N)_{\overline{1}} \simeq V_{R} \otimes_{\mathbb{C}} V_{2 N}
$$

which is provided by the invertible linear map

$$
\begin{array}{ll}
\chi(s \otimes a)(b)=s\langle a, b\rangle, & s \otimes a \in V_{R} \otimes_{\mathbb{C}} V_{2 N} \\
\chi(s \otimes a)(t)=a(s, t), & t \in V_{R}, b \in V_{2 N} .
\end{array}
$$

We say that $Q$ has rank $k$ if it can be represented as

$$
\chi^{-1}(Q)=\sum_{i=1}^{k} s_{i} \otimes a_{i},
$$

where the $s$ 's and $a$ 's are linearly independent among themselves. Of course $k \leq$ $\min (R, 2 N)$. The condition $Q^{2}=0$ can be worked out from eqs. (3.49) to be equivalent to the following constraints on the vectors $s_{i}, a_{i}$

$$
\left(s_{i}, s_{j}\right)=0, \quad\left\langle a_{i}, a_{j}\right\rangle=0
$$

for $i, j=1, \ldots, k$. These conditions are compatible with the linear independence of the $s_{i}$ and $a_{i}$ if and only if

$$
k \leq M, \quad k \leq N .
$$

This restriction on the rank $k$ allows us to define some linearly independent vectors $s_{k+1}, . ., s_{R}$ and $a_{k+1}, \ldots, a_{2 N}$ such that the matrix elements $\left(s_{i}, s_{j}\right)$, for $i, j=1, \ldots, R$ and $\left\langle a_{i}, a_{j}\right\rangle$, for $i, j=1, \ldots, 2 N$ take the form in eqs. (3.45), (3.46) and in eq. (3.47), 
respectively. Therefore, from the definition of the $\mathrm{SO}(\mathrm{R})$ and $\mathrm{SP}(2 \mathrm{~N})$ groups, there exist elements $A \in \mathrm{SO}(\mathrm{R})$ and $B \in \mathrm{SP}(2 \mathrm{~N})$ such that

$$
s_{i}=A \cdot b_{i}, \quad a_{j}=B \cdot f_{j},
$$

for $i=1, \ldots, R$ and $j=1, \ldots, 2 N$. We see that $Q$ can be brought to a simple standard form depending only on its rank $k$

$$
\chi^{-1}(\gamma(Q))=\sum_{i=1}^{k} b_{i} \otimes f_{i}
$$

by acting with the inner automorphism $\gamma=\operatorname{Ad} A^{-1} \circ \operatorname{Ad} B^{-1}$. We perform the cohomological reduction of osp $(R \mid 2 N)$ with respect to the fermionic generators

$$
\chi\left(\sum_{i=1}^{k} b_{i} \otimes f_{i}\right)
$$

by an explicit calculation. Thereby, we end up with the following statement

$$
\mathrm{H}_{Q}(\operatorname{osp}(R \mid 2 N)) \simeq \operatorname{osp}(R-2 \operatorname{rank}(Q) \mid 2 N-2 \operatorname{rank}(Q)),
$$

where $\operatorname{rank}(Q)=k \leq \min ([R / 2], N)$.

\subsection{Reduction of modules}

Let $\mathfrak{g}$ be one of the superalgebras considered in section 3.2 and $Q$ be an odd element of $\mathfrak{g}$ with vanishing self-bracket $[Q, Q]=2 Q^{2}=0$. As we have shown in section 3.2 , there is a subalgebra $\mathfrak{h} \subset \mathfrak{g}$ such that $\mathrm{H}_{Q}(\mathfrak{g}) \simeq \mathfrak{h}$.

First, notice that there is a $\mathfrak{h}$-stable filtration

$$
V \supset \operatorname{Ker}_{Q} V \supset \operatorname{Im}_{Q} V
$$

Indeed, $V$ is a $\mathfrak{h}$-submodule by restriction, while $\operatorname{Ker}_{Q} V$ and $\operatorname{Im}_{Q} V$ are $\mathfrak{h}$-submodules because $\mathfrak{h} \subset \operatorname{Ker}_{Q} \mathfrak{g}$. Finally, $\operatorname{Ker}_{Q} V \supset \operatorname{Im}_{Q} V$ follows from $Q^{2}=0$.

The existence of the $\mathfrak{h}$-stable filtration (3.57) means that $\mathrm{H}_{Q}(V)$ is generally a quotient of a submodule of the restriction of $V$ to $\mathfrak{h}$. However, if $V$ is self-dual, that is $V$ has an invariant non-degenerate scalar product, then one can repeat the steps (3.7)-(3.13), (3.17)(3.18) and prove a similar $\mathfrak{h}$-module direct sum decomposition for $V$

$$
V \simeq W \oplus E \oplus F
$$

where $W \simeq \mathrm{H}_{Q}(V)$ and $E=\operatorname{Im}_{Q} V$. We list some of the properties of the subquotients $\mathrm{H}_{Q}(V)$ that will prove useful for the following.

Lemma 2. Let $U, V$ be $\mathfrak{g}$ modules. Then the following $\mathfrak{h}$-module isomorphisms hold

a) $H_{Q}(U \oplus V) \simeq H_{Q}(U) \oplus H_{Q}(V)$ 
b) $H_{Q}\left(V^{*}\right) \simeq H_{Q}(V)^{*}$

c) $H_{Q}(U \otimes V) \simeq H_{Q}(U) \otimes H_{Q}(V)$, if $U, V$ are finite dimensional.

Proof. a) The direct sum of the modules $U$ and $V$ means that there are orthogonal idempotents $e_{U}$ and $e_{V}$ such that they commute with the action of $\mathfrak{g}$ and $e_{U} U=U$, $e_{V} V=V$. One thus has

$$
\begin{aligned}
& e_{U} \operatorname{Ker}_{Q}(U \oplus V)=\operatorname{Ker}_{Q}\left(e_{U} U \oplus e_{U} V\right)=\operatorname{Ker}_{Q} U \\
& e_{U} \operatorname{Im}_{Q}(U \oplus V)=\operatorname{Im}_{Q}\left(e_{U} U \oplus e_{U} V\right)=\operatorname{Im}_{Q} U
\end{aligned}
$$

and therefore $e_{U} \mathrm{H}_{Q}(U \oplus V)=\mathrm{H}_{Q}(U)$. Similarly, $e_{V} \mathrm{H}_{Q}(U \oplus V)=\mathrm{H}_{Q}(V)$, which completes the proof of a).

b) The elements of $\mathrm{H}_{Q}\left(V^{*}\right)$ are equivalence classes $\pi(\mu)=\mu+Q \cdot V^{*}$ of forms $\mu \in \operatorname{Ker}_{Q} V^{*}$, that is $\pi(\mu)$ is the equivalence class of forms that have the same restriction on $\operatorname{Ker}_{Q} V$ as $\mu$. Therefore the projection map $\pi$ is actually the restriction to $\operatorname{Ker}_{Q} V$. Moreover, the condition that $\mu \in \operatorname{Ker}_{Q} V^{*}$ is equivalent to the requirement that $\mu$ vanishes on $\operatorname{Im}_{Q} V$, that is $\operatorname{Ker}_{Q} V^{*} \simeq\left(V / \operatorname{Im}_{Q} V\right)^{*}$. These two observation lead to b)

$$
\begin{aligned}
\mathrm{H}_{Q}\left(V^{*}\right)=\pi\left(\operatorname{Ker}_{Q} V^{*}\right)=\left.\operatorname{Ker}_{Q} V^{*}\right|_{\operatorname{Ker}_{Q} V} & \left.\simeq\left(V / \operatorname{Im}_{Q} V\right)^{*}\right|_{\operatorname{Ker}_{Q} V} \\
& =\left(\operatorname{Ker}_{Q} V / \operatorname{Im}_{Q} V\right)^{*}=\mathrm{H}_{Q}(V)^{*}
\end{aligned}
$$

c) There exist bases $h_{a}^{\prime}, e_{i}^{\prime}, f_{i}^{\prime}$ of $U$ and $h_{b}^{\prime \prime}, e_{j}^{\prime \prime}, f_{j}^{\prime \prime}$ of $V$ that bring the action of $Q$ to a Jordan normal form

$$
\begin{array}{lll}
Q \cdot h_{a}^{\prime}=0, & Q \cdot e_{i}^{\prime}=0, & Q \cdot f_{i}^{\prime}=e_{i}^{\prime} \\
Q \cdot h_{b}^{\prime \prime}=0, & Q \cdot e_{j}^{\prime \prime}=0, & Q \cdot f_{j}^{\prime \prime}=e_{j}^{\prime \prime} .
\end{array}
$$

Computing the action of $Q$ in the corresponding tensor basis of $U \otimes V$ we get that $\operatorname{Ker}_{Q}(U \otimes V)$ is spanned by

$$
h_{a}^{\prime} \otimes h_{b}^{\prime \prime}, \quad h_{a}^{\prime} \otimes e_{j}^{\prime \prime}, \quad e_{i}^{\prime} \otimes h_{b}^{\prime \prime}, \quad e_{i}^{\prime} \otimes f_{j}^{\prime \prime}-(-1)^{\left|e_{i}^{\prime}\right|} f_{i}^{\prime} \otimes e_{j}^{\prime \prime}
$$

and $\operatorname{Im}_{Q}(U \otimes V)$ is spanned by

$$
h_{a}^{\prime} \otimes e_{j}^{\prime \prime}, \quad e_{i}^{\prime} \otimes h_{b}^{\prime \prime}, \quad e_{i}^{\prime} \otimes f_{j}^{\prime \prime}-(-1)^{\left|e_{i}^{\prime}\right|} f_{i}^{\prime} \otimes e_{j}^{\prime \prime},
$$

where $|\cdot|$ denotes the grading function. Thus, $\mathrm{H}_{Q}(U \otimes V)$ is spanned by $h_{a}^{\prime} \otimes h_{b}^{\prime \prime}$. Finally we notice that $h_{a}^{\prime}$ spans $\mathrm{H}_{Q}(U)$ and $h_{b}^{\prime \prime}$ spans $\mathrm{H}_{Q}(V)$, which proves c).

For a finite dimensional $\mathfrak{g}$-module $V$ we observe that

$$
\operatorname{sdim} \mathrm{H}_{Q}(V)=\operatorname{sdim} V .
$$

The statement follows from the existence of a Jordan normal form for the representation of $Q$ in $V$. The vanishing of the superdimension of a module $V$ is a necessary constraint for the triviality of the cohomological reduction $\mathrm{H}_{Q}(V)$. Atypical simple modules do not generally satisfy this constraint, while projective modules do, see [28]. 
Lemma 3. If $V$ is a finite dimensional projective $\mathfrak{g}$-module, then $H_{Q}(V)=0$.

Proof. Let $\Gamma^{+}$be the set of weights $\Lambda$ parametrizing the simple finite dimensional $\mathfrak{g}$-modules $S(\Lambda)$. Denote by $P(\Lambda)$ be the projective covers of $S(\Lambda)$, that is the indecomposable $\mathfrak{g}$-modules with the top $\operatorname{top}(P(\Lambda))=S(\Lambda)$. The projective module $V$ can then be represented as

$$
V \simeq \bigoplus_{\Lambda \in \Gamma^{+}} d_{\Lambda}(V) P(\Lambda)
$$

where only a finite number of multiplicities $d_{\Lambda}(V)$ do not vanish. Proving 3 becomes equivalent to proving that $\mathrm{H}_{Q}(P(\Lambda))=0$ for any $\Lambda \in \Gamma^{+}$. We show in the following that this task is equivalent to yet another one.

Define the induced modules

$$
B(\Lambda)=\operatorname{Ind}_{\mathfrak{g}_{\overline{0}}}^{\mathfrak{g}} \operatorname{Res}_{\mathfrak{g}_{\overline{0}}} S(\Lambda)=\mathfrak{U}(\mathfrak{g}) \otimes_{\mathfrak{g}_{\overline{0}}} S(\Lambda)
$$

which are finite dimensional and, according to [29], are also projective in the category of finite dimensional $\mathfrak{g}$-modules. The surjective map $\Pi: B(\Lambda) \rightarrow S(\Lambda)$

$$
\Pi\left(u \otimes_{\mathfrak{g}_{\overline{0}}} s\right)=u \cdot s
$$

defines a projective $\mathfrak{g}$-module homomorphism. By definition, top $(B(\Lambda))$ is the direct sum of all quotients of $B(\Lambda)$ by a maximal submodule. Because $B(\Lambda) / \operatorname{Ker} \Pi=S(\Lambda)$ is simple, $\operatorname{Ker} \Pi$ is a maximal submodule and therefore $S(\Lambda) \subset \operatorname{top}(B(\Lambda)$ ). On the other hand, decomposing $B(\Lambda)$ as in eq. (3.60) we explicitly compute

$$
\operatorname{top}(B(\Lambda))=\bigoplus_{\Lambda^{\prime} \in \Gamma^{+}} d_{\Lambda^{\prime}}(B(\Lambda)) \operatorname{top}\left(P\left(\Lambda^{\prime}\right)\right)=\bigoplus_{\Lambda^{\prime} \in \Gamma^{+}} d_{\Lambda^{\prime}}(B(\Lambda)) S\left(\Lambda^{\prime}\right)
$$

which from $S(\Lambda) \subset \operatorname{top}(B(\Lambda))$ implies that $P(\Lambda)$ must be a direct summand of $B(\Lambda)$. Thus, we see that proving $\mathrm{H}_{Q}(P(\Lambda))=0$ for any $\Lambda \in X^{+}$is equivalent to proving that $\mathrm{H}_{Q}(B(\Lambda))=0$ for any $\Lambda \in \Gamma^{+}$.

To compute $\mathrm{H}_{Q}(B(\Lambda))$ we construct a basis of $B(\Lambda)$ which brings the action of $Q$ to a Jordan normal form. Let $a_{1}, \ldots, a_{B}$ be a basis of $\mathfrak{g}_{\overline{0}}$ and $b_{1}, \ldots, b_{F}$ be a basis of $\mathfrak{g}_{\overline{1}}$. According to Poincaré-Birkoff-Witt theorem, the elements of the form

$$
b_{i_{1}} \cdots b_{i_{k}} a_{1}^{l_{1}} \cdots a_{B}^{l_{B}}, \quad k, l_{i} \geq 0, i_{1}<\cdots<i_{k}
$$

are a basis of $\mathfrak{U}(\mathfrak{g})$. Given a basis $s_{\alpha}$ of $S(\Lambda)$, the basis (3.64) of $\mathfrak{U}(\mathfrak{g})$ provides a basis

$$
b_{i_{1}} \cdots b_{i_{k}} \otimes s_{\alpha}, \quad k \geq 0, i_{1}<\cdots<i_{k}
$$

of $B(\Lambda)$ by means of the def. (3.61).

Choosing a basis such that $b_{1}=Q$ immediately brings the action of $Q$ to a Jordan normal form. It then becomes obvious that $\operatorname{Ker}_{Q}(B(\Lambda))=\operatorname{Im}_{Q}(B(\Lambda))$ is spanned by the basis vectors (3.65) with $i_{1}=1$. 


\subsection{Reduction of smooth functions on $G / G^{\prime}$}

We shall restrict to Lie superalgebras $\mathfrak{g}$ of the type considered in section 3.2. They all have an invariant, supersymmetric, consistent and non-degenerate bilinear form $(, \quad): \mathfrak{g} \times \mathfrak{g} \rightarrow \mathbb{C}$. Consider a subalgebra $\mathfrak{g}^{\prime}$ of $\mathfrak{g}$ such that $(, \quad)$ restricts to a nondegenerate bilinear form on $\mathfrak{g}^{\prime}$ and suppose there is an odd element $Q \in \mathfrak{g}^{\prime}$ with vanishing self-bracket $[Q, Q]=2 Q^{2}=0$.

According to eqs. (3.16), (3.18), $\mathrm{H}_{Q}(\mathfrak{g})$ and $\mathrm{H}_{Q}\left(\mathfrak{g}^{\prime}\right)$ are isomorphic to some subalgebras $\mathfrak{h} \subset \mathfrak{g}$ and, respectively, $\mathfrak{h}^{\prime} \subset \mathfrak{g}^{\prime}$, with the following direct sum decompositions

$$
\begin{aligned}
\mathfrak{g} & \simeq \mathfrak{h} \oplus \mathfrak{e} \oplus \mathfrak{f} \\
\mathfrak{g}^{\prime} & \simeq \mathfrak{h}^{\prime} \oplus \mathfrak{e}^{\prime} \oplus \mathfrak{f}^{\prime}
\end{aligned}
$$

as $\mathfrak{h}$ and $\mathfrak{h}^{\prime}$-modules, respectively. Here $\mathfrak{e}=\operatorname{Im}_{Q} \mathfrak{g}, \mathfrak{e}^{\prime}=\operatorname{Im}_{Q} \mathfrak{g}^{\prime}$. Our assumption $Q \in \mathfrak{g}^{\prime} \subset \mathfrak{g}$ implies the following subalgebra inclusions

$$
\mathfrak{h}^{\prime} \subset \mathfrak{h}, \quad \mathfrak{e}^{\prime} \subset \mathfrak{e}, \quad \mathfrak{f}^{\prime} \subset \mathfrak{f} .
$$

Let $\mathfrak{m}$ be the orthogonal complement of $\mathfrak{g}^{\prime}$ in $\mathfrak{g}$ with respect to $(, \quad)$. The assumption on the non-degeneracy of the form $(, \quad)$ and of its restriction to $\mathfrak{g}^{\prime}$ implies the following facts on $\mathfrak{m}$ :

a) $\mathfrak{m}$ is an $\mathfrak{g}^{\prime}$-module

b) $\left.(, \quad)\right|_{\mathfrak{m} \times \mathfrak{m}}$ is an $\mathfrak{g}^{\prime}$-invariant non-degenerate scalar product

c) viewed as as an $\mathfrak{g}^{\prime}$-module by restriction, $\mathfrak{g}$ decomposes as

$$
\mathfrak{g} \simeq \mathfrak{g}^{\prime} \oplus \mathfrak{m}
$$

Statements a) and b) are rather straightforward to prove, while c) results from the construction of a projection on $\mathfrak{g}^{\prime}$ with the inverted metric $(,)||_{\mathfrak{g}^{\prime} \times \mathfrak{g}^{\prime}}$, much like in eq. (3.17). From eq. (3.58) and point c), $\mathfrak{m}$ decomposes as an $\mathfrak{h}^{\prime}$-module into the direct sum

$$
\mathfrak{m} \simeq \mathfrak{n} \oplus \mathfrak{p} \oplus \mathfrak{q}
$$

where $\mathfrak{n} \simeq \mathrm{H}_{Q}(\mathfrak{m})$ and $\mathfrak{p}=\operatorname{Im}_{Q} \mathfrak{m}$. Computing the cohomology of the direct sum decomposition (3.67) with the help of property a) of lemma 2 and eqs. (3.66), (3.68) we get an analogous decomposition

$$
\mathfrak{h} \simeq \mathfrak{h}^{\prime} \oplus \mathfrak{n} .
$$

One useful consequence of eqs. (3.67)-(3.69) is the following $\mathfrak{h}^{\prime}$-module isomorphism

$$
\mathrm{H}_{Q}\left(\mathfrak{g} / \mathfrak{g}^{\prime}\right) \simeq \mathrm{H}_{Q}(\mathfrak{m}) \simeq \mathfrak{n} \simeq \mathfrak{h} / \mathfrak{h}^{\prime}
$$

Let $\mathfrak{g}_{B, \overline{0}}$ be the Grassmann envelope of $\mathfrak{g}$ with respect to some Grassmann algebra $B$ and $\mathfrak{g}_{B, \overline{0}, \dagger}$ a real form of the Lie algebra $\mathfrak{g}_{B, \overline{0}}$ with respect to a complex anti-linear involutive automorphism $\uparrow$. Suppose $G$ is a connected Lie supergroup with Lie algebra $\mathfrak{g}_{B, \overline{0}, \dagger}$ and $G^{\prime}$ 
is a connected subgroup of $G$ with Lie algebra $\mathfrak{g}_{B, \overline{0}, \dagger}^{\prime}$. Let $H$ denote the subgroup of $G$ with Lie algebra $\mathfrak{h}_{B, \overline{0}, \dagger}$ and $H^{\prime}$ the subgroup of $G^{\prime}$ with Lie algebra $\mathfrak{h}_{B, \overline{0}, \dagger}^{\prime}$. We want to perform the cohomological reduction of the space of smooth functions $\mathfrak{F}\left(G / G^{\prime}\right)$ with respect to $Q$ and show that there is an $H$-module isomorphism

$$
\mathrm{H}_{Q}\left(\mathfrak{F}\left(G / G^{\prime}\right)\right) \simeq \mathfrak{F}\left(H / H^{\prime}\right),
$$

where $\mathfrak{F}\left(H / H^{\prime}\right)$ denotes the algebra of smooth functions on $H / H^{\prime}$. Eq. (3.70) was already used in section 3.1 to give a local argument for the isomorphism (3.71). In order to prove the claim (3.71), we shall identify $\mathfrak{F}\left(G / G^{\prime}\right)$ with the space $\mathfrak{F}(G)^{G^{\prime}}$ of smooth functions on $G$ invariant with respect to the right $G^{\prime}$-action. We perform the same identification for $\mathfrak{F}\left(H / H^{\prime}\right)=\mathfrak{F}(H)^{H^{\prime}}$.

Let us look closer at $\operatorname{Im}_{Q} \mathfrak{F}\left(G / G^{\prime}\right)$. The set of points of $G / G^{\prime}$ where all elements of $\operatorname{Im}_{Q} \mathfrak{F}\left(G / G^{\prime}\right)$ vanish are precisely those points of $G / G^{\prime}$ which are invariant with respect to the action of $e^{\eta Q}$, where $\eta$ is an odd Grassmann number. We denote this subset by $\left(G / G^{\prime}\right)^{Q}$. Let $G^{Q}$ and $\left(G^{\prime}\right)^{Q}$ denote the subgroup of $G$ and, respectively, $G^{\prime}$ invariant with respect to the adjoint action of $e^{\eta Q}$. These are the subgroups on which the vector field $D(Q)$ corresponding to the adjoint action of $Q$ vanishes. This means that $\operatorname{Im}_{D(Q)} \mathfrak{F}(G)$ is the subset of smooth functions on $G$ vanishing on $G^{Q}$.

Lemma 4. The following equivalence of supermanifolds holds

$$
\left(G / G^{\prime}\right)^{Q}=G^{Q} /\left(G^{\prime}\right)^{Q}
$$

Proof. In the neighborhood of $e G^{\prime}$, where $e$ is the identity of $G$, the distinct equivalence classes of $G / G^{\prime}$ can be parametrized as

$$
e^{v} G^{\prime}
$$

where $v \in \mathfrak{m}_{B, \overline{0}, \dagger}$ is small enough. If we denote by $v$ the coordinate of the point (3.73) then we get the geodesic system of coordinates at $e G^{\prime}$. Indeed, the coordinate space $\mathfrak{m}_{B, \overline{0}, \dagger}$ can be identified with the tangent space at the point $e G^{\prime}$ with coordinates $v=0$

$$
(\mathcal{L}(v) f)(0)=\left.\frac{d}{d t}\left(e^{t v} \cdot f\right)(0)\right|_{t=0}=\left.\frac{d}{d t} f(-t v)\right|_{t=0}=-(v(f))(0),
$$

where $v \in \mathfrak{m}_{B, \overline{0}, \dagger}$ and $\mathcal{L}$ denotes the Lie derivative.

The exponential mapping

$$
v \rightarrow e^{v} G^{\prime}
$$

can be extended to the whole tangent space $\mathfrak{m}_{B, \overline{0}, \uparrow}$. This extension is in general no longer injective, that is it ceases to be a system of coordinates. However, assuming Hopf-Rinow theorem can be generalized to supermanifolds [30], the map (3.74) must be surjective, that is any group element $g \in G$ can be represented in the form

$$
g=e^{v} g^{\prime}
$$

for some $v \in \mathfrak{m}_{B, \overline{0}, \dagger}$ and $g^{\prime} \in G^{\prime}$. Using this global representation, one can easily see that $\left(G / G^{\prime}\right)^{Q}$ is the image of exponential mapping (3.74) restricted to $\operatorname{Ker}_{Q} \mathfrak{m}_{B, \overline{0}, \dagger}$. If follows that $G^{Q}$ has a transitive action on $\left(G / G^{\prime}\right)^{Q}$. Its stabilizer at $e G^{\prime} \in\left(G / G^{\prime}\right)^{Q}$ with respect to the left action on $G^{Q}$ is $\left(G^{\prime}\right)^{Q}=G^{Q} \cap G^{\prime}$. This completes the proof of claim (3.72). 
Corollary 1. Let $L(Q)$ denote the vector field corresponding to the left action of $Q$. Then one has

$$
\operatorname{Im}\left(m_{Q} \mathfrak{F}\left(G / G^{\prime}\right)=\operatorname{Im}(Q) \mathfrak{F}(G)^{G^{\prime}}=\operatorname{Im}_{D(Q)} \mathfrak{F}(G)^{G^{\prime}}=\left(\operatorname{Im}_{D(Q)} \mathfrak{F}(G)\right)^{G^{\prime}}\right.
$$

Proof. The first equality results from the identification $\mathfrak{F}\left(G / G^{\prime}\right)=\mathfrak{F}(G)^{G^{\prime}}$ while the second equality is a consequence of $Q \in \mathfrak{g}^{\prime}$. To prove the last equality notice that $\operatorname{Im}_{D(Q)} \mathfrak{F}(G)$ is composed of functions on $G$ vanishing on $G^{Q}$. Then $\left(\operatorname{Im}_{D(Q)} \mathfrak{F}(G)\right)^{G^{\prime}}$ becomes the space of functions on $G / G^{\prime}$ vanishing on the submanifold $G^{Q} / G^{\prime}$. Notice that $G^{Q} / G^{\prime}=G^{Q} /\left(G^{\prime}\right)^{Q}$, because both supermanifolds are $G^{Q}$-transitive and have the same stabilizer $\left(G^{\prime}\right)^{Q}=G^{Q} \cap G^{\prime}$. Therefore, according to eq. (3.72), $\left(\operatorname{Im}_{D(Q)} \mathfrak{F}(G)\right)^{G^{\prime}}$ can be seen as the space of functions on $G / G^{\prime}$ that vanish on $\left(G / G^{\prime}\right)^{Q}$. This, however, coincides with the definition of $\operatorname{Im}_{Q} \mathfrak{F}\left(G / G^{\prime}\right)$.

We have analogous obvious equalities for the kernel of $Q$

$$
\operatorname{Ker}_{Q} \mathfrak{F}\left(G / G^{\prime}\right)=\operatorname{Ker}_{L(Q)} \mathfrak{F}(G)^{G^{\prime}}=\operatorname{Ker}_{D(Q)} \mathfrak{F}(G)^{G^{\prime}}=\left(\operatorname{Ker}_{D(Q)} \mathfrak{F}(G)\right)^{G^{\prime}}
$$

Combining eqs. (3.75), (3.76) we get the following prescription for computing the cohomology

$$
\mathrm{H}_{Q}\left(\mathfrak{F}\left(G / G^{\prime}\right)\right)=\left(\mathrm{H}_{D(Q)}(\mathfrak{F}(G))\right)^{G^{\prime}}
$$

Let us now concentrate on computing $\mathrm{H}_{D(Q)}(\mathfrak{F}(G))$.

The image of a function $f$ under the projection map $\pi: \mathfrak{F}(G) \rightarrow \mathfrak{F}(G) / \operatorname{Im}_{D(Q)} \mathfrak{F}(G)$ given by

$$
\pi(f)=f+\operatorname{Im}_{D(Q)} \mathfrak{F}(G)
$$

is the equivalence class of functions which have the same restriction on $G^{Q}$ as $f$, that is

$$
\pi(f)=\left.f\right|_{G^{Q}}
$$

In particular any function whose restriction to $G^{Q}$ vanishes must be in the image of $Q$.

Notice that the left and the right $G$-actions on $\mathfrak{F}(G)$ induce corresponding left and right $G^{Q}$-action on the quotient space $\mathfrak{F}(G) / \operatorname{Im}_{D(Q)} \mathfrak{F}(G)$

$$
L(X) \pi(f):=\pi(L(X) f), \quad R(X) \pi(f):=\pi(R(X) f) .
$$

Lemma 5. The following isomorphism of $H$-modules and commutative algebras holds

$$
H_{D(Q)}(\mathfrak{F}(G))^{G^{\prime}} \simeq \mathfrak{F}\left(H / H^{\prime}\right)
$$

Proof. If $X \in \mathfrak{g}$ and $f \in \operatorname{Ker}_{L(Q)} \mathfrak{F}(G)^{G^{\prime}}$, then

$$
L([Q, X]) \pi(f)=\pi(L[Q, X] f)=\pi(L(Q) L(X) f)=\pi(D(Q) L(X) f)=0,
$$

because the left and right $\mathfrak{g}$ actions on $\mathfrak{F}(G)$ commute and $\operatorname{Ker} \pi=\operatorname{Im}_{D(Q)} \mathfrak{F}(G)$. This shows that the space of functions $\mathrm{H}_{D(Q)}(\mathfrak{F}(G))^{G^{\prime}}$ is left invariant with respect to the action of $\mathfrak{e}$. Denote by $N$ the subgroup of $G$ with the Lie superalgebra $\mathfrak{e}$. The latter being an 
ideal of $\operatorname{Ker}_{Q} \mathfrak{g}, N$ is a normal subgroup of $G^{Q}$ with $H=N \backslash G^{Q}$. Then eq. (3.80) claims that $\mathrm{H}_{D(Q)}(\mathfrak{F}(G))^{G^{\prime}}$ is a space of functions on $N \backslash G^{Q} / G^{\prime}=H / G^{\prime}=H / H^{\prime}$. The last equality comes from the fact that both $H / G^{\prime}$ and $H / H^{\prime}$ are $H$-transitive and have the same stabilizer $H^{\prime}=G^{\prime} \cap H$.

In conclusion we wee that the cohomology of a smooth function on $G / G^{\prime}$ is computed by restricting it to $H / H^{\prime} \subset G / G^{\prime}$. We denote this restriction map by $\rho$.

\subsection{Reduction of smooth tensor forms on $G / G^{\prime}$}

Let $T_{k}\left(G / G^{\prime}\right)$ be the space of smooth tensor forms of rank $k$ on $G / G^{\prime}$. We claim that eq. (3.71) can be generalized to

$$
\mathrm{H}_{Q}\left(T_{k}\left(G / G^{\prime}\right)\right) \simeq T_{k}\left(H / H^{\prime}\right)
$$

where $T_{k}\left(H / H^{\prime}\right)$ is the space of smooth tensor forms of rank $k$ on $H / H^{\prime}$. We shall only give a local argument. Introducing the geodesic coordinates (3.73), one can perform the following identification in the neighborhood of the point $e G^{\prime} \in G / G^{\prime}$

$$
T_{k}\left(G / G^{\prime}\right) \simeq \mathfrak{F}\left(G / G^{\prime}\right) \otimes \mathfrak{m}^{\otimes k}
$$

This local trivialization extends to an isomorphism of $G^{\prime}$-modules. Using the property c) of lemma 2, we get

$$
\mathrm{H}_{Q}\left(T_{k}\left(G / G^{\prime}\right)\right) \simeq \mathrm{H}_{Q}\left(\mathfrak{F}\left(G / G^{\prime}\right)\right) \otimes \mathrm{H}_{Q}(\mathfrak{m})^{\otimes k} \simeq \mathfrak{F}\left(H / H^{\prime}\right) \otimes \mathfrak{n}^{\otimes k} \simeq T_{k}\left(H / H^{\prime}\right) .
$$

Most probably, one can give a global argument for the claim (3.81) by introducing the frame bundle

$$
T_{k}\left(G / G^{\prime}\right) \simeq\left(\mathfrak{F}(G) \otimes F(G)^{\otimes k}\right)^{G^{\prime}},
$$

where $F(G)$ is the moving frame attached to every point of $G$, which is built out of the components of the Maurer-Cartan form.

In conclusion, the cohomology of a tensor form on $G / G^{\prime}$ is computed, as can be seen from eq. (3.82), by restricting it i) to the submanifold $H / H^{\prime}$ and ii) to the tensor space of $H / H^{\prime}$. Step ii) is equivalent to throwing out all components of the tensor not lying in the tensor space of $H / H^{\prime}$ seen as a submanifold of $G / G^{\prime}$. We denote this restriction map by $\rho$ again.

\subsection{Reduction of $L_{2}\left(G / G^{\prime}\right)$}

We want to refine (3.71) and show that the elements of $\mathrm{H}_{Q}\left(L_{2}\left(G / G^{\prime}\right)\right)$ are square integrable with respect to some $H$-invariant measure on $H / H^{\prime}$, that is

$$
\mathrm{H}_{Q}\left(L_{2}\left(G / G^{\prime}\right)\right) \simeq L_{2}\left(H / H^{\prime}\right) .
$$

In order to do so, introduce the geodesic coordinates $v$ of eq. (3.73). Let $v_{\mathfrak{n}}, v_{\mathfrak{p}}$ and $v_{\mathfrak{q}}$ denote the projection of $v$ onto the real Grassmann envelope of the direct summand $\mathfrak{n}, \mathfrak{p}$ 
and, respectively, $\mathfrak{q}$ in eq. (3.68). We then embed $\mathfrak{F}\left(H / H^{\prime}\right)$ into $\mathfrak{F}\left(G / G^{\prime}\right)$ by means of the injection map

$$
i(f)(v)=f\left(v_{\mathfrak{n}}\right) e^{\alpha\left(v_{\mathfrak{p}}, v_{\mathfrak{q}}\right)},
$$

where $v$ is small enough and $\alpha$ is, for the moment, an arbitrary number. Notice that eq. (3.84) defines the function $i(f)$ globally. Indeed, the definition (3.84) allows to compute the action of the enveloping Lie superalgebra $\mathfrak{U}(\mathfrak{g})$ on $i(f)$. The latter can be extended to the action of the group $G$, whose knowledge is enough to define the values of $i(f)$ at any point of $G / G^{\prime}$.

The most important property of the injection map (3.84) is

$$
\pi \circ i=\mathbb{1},
$$

where $\pi$ is the projection of eqs. (3.78), (3.79). As a consequence, any element of $\operatorname{Ker}_{Q} \mathfrak{F}\left(G / G^{\prime}\right)$ can be represented in the form

$$
i(f)+\mathcal{L}(Q) h,
$$

where $\mathcal{L}(Q)$ denotes the Lie derivative with respect to $Q$.

We now prove (3.83) by showing that for a proper choice of $\alpha$ in eq. (3.84) one has

$$
\left\langle i\left(f_{1}\right), i\left(f_{2}\right)\right\rangle_{G / G^{\prime}}=\left\langle f_{1}, f_{2}\right\rangle_{H / H^{\prime}} .
$$

The equation should be understood as follows: i) the existence of one side implies the existence of the other side and ii) for a $G$-invariant scalar product $\langle,\rangle_{G / G^{\prime}}$ on $L_{2}\left(G / G^{\prime}\right)$ induced by the $G$-invariant measure on $G / G^{\prime}$ there is a corresponding $H$-invariant scalar product $\langle,\rangle_{H / H^{\prime}}$ on $L_{2}\left(H / H^{\prime}\right)$ induced by the $H$-invariant measure on $H / H^{\prime}$.

Indeed, let the measure on $G / G^{\prime}$ be given locally by $d \mu_{G}(v)=w(v) d v$. Suppose $i(f)$ is $L_{2}$ normalizable. Then its norm can be written in the form

$$
\int_{H / H^{\prime}} d \mu_{H} f^{2}
$$

where $d \mu_{H}$ is a measure on $H / H^{\prime}$ locally defined by a weight function $w^{\prime}\left(v_{\mathfrak{n}}\right)$ obtained by integrating

$$
w(v) e^{2 \alpha\left(v_{\mathfrak{p}}, v_{\mathfrak{q}}\right)},
$$

with respect to the coordinates $v_{\mathfrak{p}}$ and $v_{\mathfrak{q}}$. Notice that there is always a choice of $\alpha$ such that $w^{\prime}$ exists even for non-compact homogeneous spaces $G / G^{\prime}$. Of course, in order to perform the integration yielding the explicit form of $w^{\prime}$ one must work with an atlas of $G / G^{\prime}$. However, the only thing that matters to us is its $H$-invariance or, equivalently, the $H$-invariance of the scalar product $\langle,\rangle_{H / H^{\prime}}$ associated to it by eq. (3.87). We thus check

$$
\left\langle i\left(\mathcal{L}(X) f_{1}\right), i\left(f_{2}\right)\right\rangle_{G / G^{\prime}}+\left\langle i\left(f_{1}\right), i\left(\mathcal{L}(X) f_{2}\right)\right\rangle_{G / G^{\prime}}=0, \quad X \in \mathfrak{h} .
$$

Notice that $\left(v_{\mathfrak{p}}, v_{\mathfrak{q}}\right)$ is $Q$-exact because its restriction to $v_{\mathfrak{q}}=0$ vanishes. Therefore $\mathcal{L}(X)\left(v_{\mathfrak{p}}, v_{\mathfrak{q}}\right)$ is also $Q$-exact, because $[Q, X]=0$. Finally,

$$
(\mathcal{L}(X) i(f))(v)-i(\mathcal{L}(X) f)(v)=f\left(v_{\mathfrak{n}}\right) \alpha e^{\alpha\left(v_{\mathfrak{p}}, v_{\mathfrak{q}}\right)} \mathcal{L}(X)\left(v_{\mathfrak{p}}, v_{\mathfrak{q}}\right)
$$


is $Q$-exact as well, because $f\left(v_{\mathfrak{n}}\right) e^{\alpha\left(v_{\mathfrak{p}}, v_{\mathfrak{q}}\right)}$ is $Q$-invariant. We then use the exactness of the expression (3.89) to commute the Lie derivative $\mathcal{L}(X)$ with the injection $i$ in eq. (3.88).

We conclude this section by noticing that eq. (3.87) can be written in an equivalent way as

$$
\left\langle f_{1}, f_{2}\right\rangle_{G / G^{\prime}}=\left\langle\rho\left(f_{1}\right), \rho\left(f_{2}\right)\right\rangle_{H / H^{\prime}},
$$

where $f_{1}, f_{2} \in \operatorname{Ker}_{Q} L_{2}\left(G / G^{\prime}\right)$. This is the localization phenomenon.

\section{Cohomological reduction in the field theory}

We are now prepared to revisit the sigma models on $G / G^{\prime}$. We have shown in section 2.3 how the local observables of the sigma model on $G / G^{\prime}$ can be constructed from functions on $L_{2}\left(G / G^{\prime}\right)$ and (some well behaved subspace of the space of smooth) tensor forms on $G / G^{\prime}$. The results of section (3.4)-(3.6) straightforwardly imply that the cohomological reduction of the space of local observables in the sigma model on $G / G^{\prime}$ coincides precisely with the space of local observables in the sigma model on $H / H^{\prime}$, that is

$$
\mathrm{H}_{Q}\left(\mathcal{F}_{G / G^{\prime}}\right) \simeq \mathcal{F}_{H / H^{\prime}}
$$

We now look at correlation functions of local fields $\mathcal{O}$ that are $Q$-invariant. As the results of the previous section suggest, we shall demonstrate that any correlation function of such fields can be computed in the $H / H^{\prime}$ coset superspace theory.

First we need to compute the cohomological reduction of the action $\mathcal{S}_{G / G^{\prime}}$ associated to the Lagrangian in eq. (2.2). Since the Lagrangian is entirely fixed by a $G$-invariant metric and a $G$-invariant 2-form, we can apply the results of section 3.5 in order to compute their cohomology class. The classes of the two tensor forms are computed by restricting them to the points of the submanifold $H / H^{\prime}$ and to its tensor space respectively. As a result we obviously get an $H$-invariant metric and an exact $H$-invariant 2-form on $H / H^{\prime}$. Employing the restriction map $\rho$ of section (3.4), (3.5), we conclude that

$$
\rho\left(\mathcal{S}_{G / G^{\prime}}\right)=\mathcal{S}_{H / H^{\prime}}
$$

is an action for the sigma model on $H / H^{\prime}$ with a similar kinetic term and $B$-field structure as $\mathcal{S}_{G / G^{\prime}}$. The pullback of eq. (4.2) takes a more familiar form to usual cohomological calculations in field theory

$$
\mathcal{S}_{G / G^{\prime}}=\mathcal{S}_{H / H^{\prime}}+\mathcal{L}(Q) R
$$

where $\mathcal{L}(Q)$ denotes the Lie derivative with respect to $Q$ and $R$ is some residual functional, obviously non $G$-invariant. The possibility of constructing $G$-invariant terms $\mathcal{L}(Q) R$ out of non $G$-invariant terms $R$ is a special feature of the supergroup symmetry. According to one of the main ideas behind cohomological reduction, the $Q$-exact term in the action does not contribute to the calculation of correlation functions of $Q$-invariant local fields.

To make things more precise, notice that the localization formula (3.90) for the computation of the scalar product of $Q$-invariant functions can be generalized to the integral 
of any $Q$-invariant object. Therefore, we trivially obtain from eq. (2.16)

$$
\begin{aligned}
\left\langle\prod_{i=1}^{N} \mathcal{O}_{i}\left(x_{i}\right)\right\rangle_{G / G^{\prime}} & =\int_{\mathcal{H}} \mathrm{d} \mu_{H} e^{-\rho\left(\mathcal{S}_{G / G^{\prime}}\right)} \prod_{i=1}^{N} \rho\left(\mathcal{O}_{i}\right)\left(x_{i}\right) \\
& =\left\langle\prod_{i=1}^{N} \rho\left(\mathcal{O}_{i}\right)\left(x_{i}\right)\right\rangle_{H / H^{\prime}} .
\end{aligned}
$$

where we have used eq. (4.2). Consequently, the subsector of the sigma model on $G / G^{\prime}$ which we obtain through cohomological reduction is composed of the localized observables $\rho\left(\mathcal{O}_{i}\right)$. Finally, using the central statement (4.3), we conclude that this subsector is exactly identified with the local observables of the sigma model on $H / H^{\prime}$.

\section{Applications}

In the first subsection we discuss applications of cohomological reduction to conformal field theory. In the second subsection we present a general treatment of sigma models on supercoset spaces $G / G^{\mathbb{Z}_{2}}$ defined by a degree two automorphism, that is on symmetric superspaces. The last subsection deals with some specific examples involving automorphisms of degree four.

\subsection{Conformal field theory}

The cohomological reduction we have described in the previous two subsections allows us to identify certain simple subsectors of the parent theory in which all correlation functions can be computed explicitly through the reduced model. The latter is often much simpler than the original theory. In fact, we shall find many examples below in which the subsector is a free or even topological field theory. The existence of such simple subsectors may signal very special features of the parent model. In particular, it can imply its scale invariance.

In order to make a more precise statement we need a bit of preparation. Let us recall that the coset $G / G^{\prime}$ gives rise to a family of sigma models which is parametrized by the metric $\mathrm{G}$ and the $B$-field B. $G$-invariance of the action determines the two background fields up to a finite number of parameters. Upon quantization, these parameters may be renormalized. This renormalization of $\mathrm{G}$ and $\mathrm{B}$ can affect the properties of our theory and in particular of its stress tensor.

Let us now consider the quantized $G / G^{\prime}$ model that comes with some fixed choice of $\mathrm{G}$ and B. The associated stress tensor $T_{G}$ is conserved and symmetric. On the other hand, the trace of $T_{G}$ may be non-zero due to quantum effects. The components of $T_{G}$ are $G$ invariant, i.e. they commute with all generators $X \in \mathfrak{g}$. In general, $T_{G}$ can be decomposed into a sum $T_{G}=\sum_{i} T_{G}^{(i)}$ of terms where each of the summands $T_{G}^{(i)}$ belongs to a single indecomposable representation of $\mathfrak{g}$. We say that $T_{G}$ is a true $G$-invariant if every summand $T_{G}^{(i)}$ is a direct summand. This must be distinguished from more generic cases for which some of the summands $T_{G}^{(i)}$, although transforming in the trivial representation of $\mathfrak{g}$, are coupled to other fields through the action of a nilpotent symmetry generator $N$ from the 


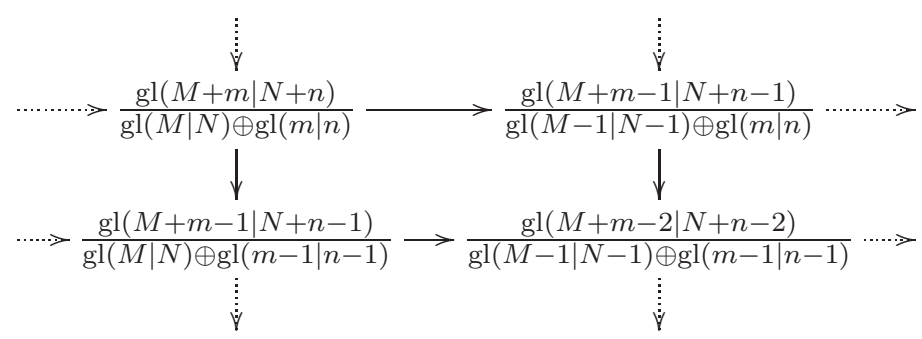

Figure 1. Possible cohomological reductions of $\operatorname{gl}(M+m \mid N+n) / \operatorname{gl}(M \mid N) \oplus \operatorname{gl}(m \mid n)$.

center of the enveloping Lie superalgebra $\mathfrak{U}(\mathfrak{g})$. In this case, $T_{G}^{(i)}=N t_{G}^{(i)}$ for some field $t_{G}^{(i)}$, which is called a logarithmic partner of $T_{G}^{(i)}$.

Let us now assume that the tensor $T_{G}$ is a true $G$-invariant in the sense we have described above. Suppose furthermore that the theory contains a conformal subsector $H / H^{\prime}$ with a non-vanishing stress tensor $T_{H}$. According to our assumption, $T_{H}$ is conserved, symmetric and traceless. Consequently, the stress tensor of the original theory must be conserved, symmetric and traceless up to some $Q$-exact terms. Since we assumed $T_{G}$ to be a true invariant, though, non of its components - and in particular the trace of $T_{G}$ can be obtained by acting with an element of $\mathfrak{U}(\mathfrak{g})$ on some other fields. Hence, $T_{G}$ must be traceless and hence the $G / G^{\prime}$ model is conformal.

Let us stress again that our assumption on $T_{G}$ to be a true invariant is rather strong. We are not prepared to state precise conditions under which this assumption is actually satisfied in general. However, when the superspaces $G / G^{\prime}$ have at most one degree of freedom in the choice of $\mathrm{G}$ and $\mathrm{B}$ one can get a simple constraint for the conformality of the parent theory from the conformality of the cohomological subsector theory: the sigma model $G / G^{\prime}$ is conformal if $H / H^{\prime}$ is conformal and its central charge is non-zero. Indeed, in this case $\mathrm{G}$ and $B$ is either proportional to i) a single $\mathfrak{g}$ true invariant or to ii) a single invariant socle of a $\mathfrak{g}$-indecomposable module. If $H / H^{\prime}$ is the conformally invariant maximal cohomological reduction with a non-zero central charge, then $T_{G}$ cannot be an invariant socle. Otherwise we would get a contradiction, because its 2-point function would vanish and the 2-point function of $T_{G}$ must coincide with the 2-point function of $T_{H}$. The latter, however, cannot vanish because the central charge of the conformal $H / H^{\prime}$ sigma model is non-zero.

\subsection{Sigma models on symmetric superspaces}

In this section, we want to present a classification of the cohomological reductions of $\mathbb{Z}_{2}$ cosets, i.e. of symmetric superspaces. These supermanifolds $G / G^{\prime}$ have the property that $G^{\prime}$ is a direct product of supergroups of which at most two are simple. For each simple factor whose superalgebra contains nilpotent fermionic elements, we can perform the cohomological reduction. Reductions performed with $Q$ operators that come from different simple factors commute with each other. As an example, consider the coset space $\mathfrak{g} / \mathfrak{g}^{\prime}=\operatorname{gl}(M+m \mid N+n) / \mathrm{gl}(M \mid N) \oplus \operatorname{gl}(m \mid n)$. The denominator has two simple factors, so that we can reduce in two ways as outlined in figure 1. 


\begin{tabular}{|c|c|c|}
\hline $\mathcal{R}$ & $\mathcal{M}$ & Comments \\
\hline$\frac{\operatorname{psl}(1 \mid 1) \oplus \operatorname{psl}(1 \mid 1)}{\operatorname{psl}(1 \mid 1)}$ & $\frac{\operatorname{psl}(1+\alpha \mid 1+\alpha) \oplus \operatorname{psl}(1+\alpha \mid 1+\alpha)}{\operatorname{psl}(1+\alpha \mid 1+\alpha)}$ & $\mathrm{C}$ \\
\hline$\frac{\operatorname{gl}(1 \mid 1) \oplus \operatorname{gl}(1 \mid 1)}{\operatorname{gl}(1 \mid 1)}$ & $\frac{\operatorname{gl}(1+\alpha \mid 1+\alpha) \oplus \operatorname{gl}(1+\alpha \mid 1+\alpha)}{\operatorname{gl}(1+\alpha \mid 1+\alpha)}$ & $\mathrm{T}$ \\
\hline$\frac{\mathrm{sl}(\mathrm{R}) \oplus \mathrm{sl}(\mathrm{R})}{\mathrm{sl}(\mathrm{R})}$ & $\frac{\operatorname{sl}(R+\alpha \mid \alpha) \oplus \operatorname{sl}(R+\alpha \mid \alpha)}{\operatorname{sl}(R+\alpha \mid \alpha)}$ & \\
\hline$\frac{\mathrm{gl}(\mathrm{R}+\mathrm{S})}{\mathrm{gl}(\mathrm{R}) \oplus \operatorname{gl}(\mathrm{S})}$ & $\frac{\operatorname{gl}(R+S+\alpha+\beta \mid \alpha+\beta)}{\operatorname{gl}(R+\alpha \mid \alpha) \oplus \operatorname{gl}(S+\beta \mid \beta)}$ & $\mathrm{T}$ for $R=0$ or $S=0$ \\
\hline $\operatorname{gl}(R \mid S)$ & $\operatorname{gl}(R+\alpha+\beta \mid S+\alpha+\beta)$ & $\mathrm{C}$ for $R=S=1$ \\
\hline$\overline{\mathrm{gl}(\mathrm{R}) \oplus \mathrm{gl}(\mathrm{S})}$ & $\overline{\operatorname{gl}(R+\alpha \mid \alpha) \oplus \operatorname{gl}(\beta \mid S+\beta)}$ & $\mathrm{T}$ for $R=0$ or $S=0$ \\
\hline$\frac{\operatorname{psl}(1 \mid 1) \oplus \operatorname{psl}(1 \mid 1)}{\operatorname{psl}(1 \mid 1)}$ & $\frac{\operatorname{psl}(2 \alpha \mid 2 \alpha)}{\operatorname{osp}(2 \alpha \mid 2 \alpha)}$ & $\mathrm{C}$ \\
\hline$\frac{\operatorname{gl}(2 \mid 2)}{\operatorname{osp}(2 \mid 2)}$ & $\frac{\operatorname{gl}(2+2 \alpha \mid 2+2 \alpha)}{\operatorname{osp}(2+2 \alpha \mid 2+2 \alpha)}$ & $\mathrm{T}$ \\
\hline$\frac{\mathrm{sl}(\mathrm{R})}{\mathrm{so}(\mathrm{R})}$ & $\frac{\operatorname{sl}(R+2 \alpha \mid 2 \alpha)}{\operatorname{osp}(R+2 \alpha \mid 2 \alpha)}$ & $\mathrm{T}$ for $R=1$ \\
\hline$\frac{\mathrm{sl}(2 \mathrm{R})}{\mathrm{sp}(2 \mathrm{R})}$ & $\frac{\operatorname{sl}(2 \alpha \mid 2 R+2 \alpha)}{\operatorname{osp}(2 \alpha \mid 2 R+2 \alpha)}$ & \\
\hline$\frac{\operatorname{sl}(1 \mid 2 R)}{\operatorname{osp}(1 \mid 2 R)}$ & $\frac{\operatorname{sl}(1+2 \alpha \mid 2 R+2 \alpha)}{\operatorname{osp}(1+2 \alpha \mid 2 R+2 \alpha)}$ & \\
\hline$\frac{\mathrm{so}(\mathrm{R}) \oplus \mathrm{so}(\mathrm{R})}{\mathrm{so}(\mathrm{R})}$ & $\frac{\operatorname{osp}(R+2 \alpha \mid 2 \alpha) \oplus \operatorname{osp}(R+2 \alpha \mid 2 \alpha)}{\operatorname{osp}(R+2 \alpha \mid 2 \alpha)}$ & $\begin{array}{l}\mathrm{C} \text { for } R=2 \\
\mathrm{~T} \text { for } R=0 \text { or } R=1\end{array}$ \\
\hline$\frac{\mathrm{sp}(2 \mathrm{R}) \oplus \mathrm{sp}(2 \mathrm{R})}{\mathrm{sp}(2 \mathrm{R})}$ & $\frac{\operatorname{osp}(2 \alpha \mid 2 R+2 \alpha) \oplus \operatorname{osp}(2 \alpha \mid 2 R+2 \alpha)}{\operatorname{osp}(2 \alpha \mid 2 R+2 \alpha)}$ & $\mathrm{T}$ for $R=0$ \\
\hline$\frac{\operatorname{osp}(1 \mid 2 R) \oplus \operatorname{osp}(1 \mid 2 R)}{\operatorname{osp}(1 \mid 2 R)}$ & $\frac{\operatorname{osp}(1+2 \alpha \mid 2 R+2 \alpha) \oplus \operatorname{osp}(1+2 \alpha \mid 2 R+2 \alpha)}{\operatorname{osp}(1+2 \alpha \mid 2 R+2 \alpha)}$ & \\
\hline$\frac{\mathrm{so}(\mathrm{R}+\mathrm{S})}{\mathrm{so}(\mathrm{R}) \oplus \mathrm{so}(\mathrm{S})}$ & $\frac{\operatorname{osp}(R+S+2 \alpha+2 \beta \mid 2 \alpha+2 \beta)}{\operatorname{osp}(R+2 \alpha \mid 2 \alpha) \oplus \operatorname{osp}(S+2 \beta \mid 2 \beta)}$ & $\begin{array}{l}\mathrm{C} \text { for } R=S=1 \\
\mathrm{~T} \text { for } R=0 \text { or } S=0\end{array}$ \\
\hline$\frac{\mathrm{osp}(R \mid 2 S)}{\operatorname{so}(\mathrm{R}) \oplus \operatorname{sp}(2 \mathrm{~S})}$ & $\frac{\operatorname{osp}(R+2 \alpha+2 \beta \mid 2 S+2 \alpha+2 \beta)}{\operatorname{osp}(R+2 \alpha \mid 2 \alpha) \oplus \operatorname{osp}(2 \beta \mid 2 S+2 \beta)}$ & $\mathrm{T}$ for $R=0$ or $S=0$ \\
\hline$\frac{\mathrm{sp}(2 \mathrm{R}+2 \mathrm{~S})}{\mathrm{sp}(2 \mathrm{R}) \oplus \mathrm{sp}(2 \mathrm{~S})}$ & $\frac{\operatorname{osp}(2 \alpha+2 \beta \mid 2 R+2 S+2 \alpha+2 \beta)}{\operatorname{osp}(2 \alpha \mid 2 R+2 \alpha) \oplus \operatorname{osp}(2 \beta \mid 2 S+2 \beta)}$ & $\mathrm{T}$ for $R=0$ or $S=0$ \\
\hline$\frac{\operatorname{osp}(2 \mid 2 R+2 S)}{\operatorname{osp}(1 \mid 2 R) \oplus \operatorname{osp}(1 \mid 2 S)}$ & $\frac{\operatorname{osp}(2+2 \alpha+2 \beta \mid 2 R+2 S+2 \alpha+2 \beta)}{\operatorname{osp}(1+2 \alpha \mid 2 R+2 \alpha) \oplus \operatorname{osp}(1+2 \beta \mid 2 S+2 \beta)}$ & \\
\hline$\frac{\mathrm{so}(2 \mathrm{R})}{\mathrm{gl}(\mathrm{R})}$ & $\frac{\operatorname{osp}(2 R+2 \alpha \mid 2 \alpha)}{\operatorname{gl}(R+\alpha \mid \alpha)}$ & $\mathrm{T}$ for $R=0,1$ \\
\hline$\frac{\mathrm{sp}(2 \mathrm{R})}{\mathrm{gl}(\mathrm{R})}$ & $\frac{\operatorname{osp}(2 \alpha \mid 2 R+2 \alpha)}{\operatorname{gl}(\alpha \mid R+\alpha)}$ & $\mathrm{T}$ for $R=0$ \\
\hline
\end{tabular}

Table 2. The left column presents the possible minimal non-trivial sectors labelled by $R, S$ and the right one the chain of models to which they belong. We denote by $\mathrm{T}$ the models that have a topological subsector and by $\mathrm{C}$ those models that are conformally invariant.

In table 2 below, we describe the different cohomological sectors of all possible sigma models on symmetric superspaces. We only write down the complex case, but different reality conditions can then easily be taken into consideration.

Some of the minimal subsectors are topological. This occurs when the whole Lagrangian is in the image of $Q$, which is the case whenever the right side of table (2) can be brought to the form $\mathfrak{g} / \mathfrak{g}$. This happens for the GL $(\mathrm{N} \mid \mathrm{N}) \operatorname{OSP}(2 \mathrm{~N}+1 \mid 2 \mathrm{~N})$ and 
OSP $(2 \mathrm{~N} \mid 2 \mathrm{~N})$ principal chiral models as well as for the cosets

$$
\frac{\mathrm{GL}(\mathrm{N}+\mathrm{p} \mid \mathrm{N}+\mathrm{q})}{\mathrm{GL}(\mathrm{N} \mid \mathrm{N}) \times \mathrm{GL}(\mathrm{p} \mid \mathrm{q})} \quad \frac{\mathrm{GL}(2 \mathrm{~N} \mid 2 \mathrm{~N})}{\operatorname{OSP}(2 \mathrm{~N} \mid 2 \mathrm{~N})} \quad \frac{\mathrm{OSP}(2 \mathrm{~N}+\mathrm{p} \mid 2 \mathrm{~N}+2 \mathrm{q})}{\operatorname{OSP}(2 \mathrm{~N} \mid 2 \mathrm{~N}) \times \operatorname{OSP}(\mathrm{p} \mid 2 \mathrm{q})} \quad \frac{\mathrm{OSP}(2 \mathrm{~N} \mid 2 \mathrm{~N})}{\mathrm{GL}(\mathrm{N} \mid \mathrm{N})} .
$$

On the other hand, some cohomological reductions lead to free conformal field theories, for which there are only two possibilities. Either they reduce to the $c=1$ free boson model to the $c=-2$ theory of a pair of symplectic fermions. The former case occurs for the OSP $(2 \mathrm{~N}+2 \mid 2 \mathrm{~N})$ principal chiral model and the real Grassmannians

$$
\frac{\operatorname{OSP}(2+2 \mathrm{~m}+2 \mathrm{n} \mid 2 \mathrm{~m}+2 \mathrm{n})}{\operatorname{OSP}(1+2 \mathrm{~m} \mid 2 \mathrm{~m}) \times \operatorname{OSP}(1+2 \mathrm{n} \mid 2 \mathrm{n})},
$$

whereas the latter occurs for the PSL $(\mathrm{N} \mid \mathrm{N})$ principal chiral model as well as for the cosets

$$
\frac{\mathrm{GL}(\mathrm{m}+\mathrm{n}+1 \mid \mathrm{m}+\mathrm{n}+1)}{\mathrm{GL}(\mathrm{m}+1 \mid \mathrm{m}) \times \mathrm{GL}(\mathrm{n} \mid \mathrm{n}+1)} \quad \frac{\operatorname{PSL}(2 \mathrm{~N} \mid 2 \mathrm{~N})}{\operatorname{OSP}(2 \mathrm{~N} \mid 2 \mathrm{~N})} .
$$

As was shown in [22] by direct computation of the all loop $\beta$ function, these are the only sigma models on symmetric spaces that are conformally invariant. The superspaces $G / G^{\prime}$ in eqs (5.1), (5.2) have only one radius and no $G$-invariant $B$-field. We thus see that the argument of section 5.1 leads to the same classification of conformally invariant sigma models, while this time being non-perturbative in nature.

\subsection{Examples involving generalized symmetric spaces}

We will now turn our attention to a few generalized symmetric spaces in which the denominator supergroup $G^{\prime}$ is left invariant under the action of some automorphism $\Omega: G \mapsto G$ of order four. We are not attempting to provide a classification of such cosets, but restrict our discussion to three interesting examples. The first series of models contains theories whose minimal subsector is given by the sigma model for $A d S_{5} \times S^{5}$ and $A d S_{2} \times S^{2}$ spaces. The second and third example extend the construction of superspheres and complex projective spaces, respectively. In all three families of models we shall identify previously unknown candidates for conformal cosets, see eqs. (5.6), (5.11) and (5.14).

Example 1. We look at the coset

$$
\mathfrak{g} / \mathfrak{g}^{\prime}=\frac{\operatorname{psu}(2(M+m) \mid 2(N+n))}{\operatorname{osp}(2 m \mid 2 n) \oplus \operatorname{osp}(2 N \mid 2 M)}
$$

defined for $M+m=N+n$ by the following automorphism of order four: $\Omega=-s t \circ \operatorname{Ad}_{X} \circ$ $\operatorname{Ad}_{Y}$ with

$$
X=\left(\begin{array}{l|l}
\mathbb{1}_{M+m} & \\
\hline & \mathbb{1}_{M+n} \mathbb{1}_{N+n}
\end{array}\right) \quad Y=\operatorname{diag}\left(\mathbb{1}_{m},-\mathbb{1}_{2 M+m}, \mathbb{1}_{N+2 n},-\mathbb{1}_{N}\right) .
$$

Here, in order to properly define the automorphism, one has to embed the superalgebra $\operatorname{psu}(2(M+m) \mid 2(N+n))$ in the fundamental representation of $\mathrm{su}(2(M+m) \mid 2(N+n))$. 
The invariant subalgebra $\mathfrak{g}^{\prime}$ is a direct sum for which the grading of the second summand is opposite that of the first one. In order to know the number of free parameters in the metric and $B$ field defining the model, we have to know how the $\Omega$ eigenspaces transform under the action of $\mathfrak{g}^{\prime}$. The result is

$$
\mathfrak{m}_{1} \cong \square \otimes \square \quad \mathfrak{m}_{2} \cong(\varnothing \otimes \square) \oplus(\square \otimes \varnothing) \quad \mathfrak{m}_{3} \cong \square \otimes \square
$$

Here, as well as in the following examples, $\varnothing$ denotes the trivial representation, the fundamental representation and $\square^{*}$ its dual. Tensor products of the fundamental representation and of its dual that possess certain permutation symmetry are denoted by the appropriate Young tableaux.

We want to mention three special cases for these cosets

- Without loss of generality, we choose $Q$ to lie only in the second direct summand of $\mathfrak{g}^{\prime}$. Assuming that $M=N$ and thus $m=n$, we see that the maximal reduction in this case leads to the sigma model on the $\mathbb{Z}_{2}$ coset PSU $(2 \mathrm{~m} \mid 2 \mathrm{~m}) / \mathrm{OSP}(2 \mathrm{~m} \mid 2 \mathrm{~m})$, which is conformal. We thus arrive at the conclusion that the sigma models on the $\mathbb{Z}_{4}$ coset spaces

$$
\mathcal{C}_{(N, n)} \cong \frac{\operatorname{PSU}(2(\mathrm{~N}+\mathrm{n}) \mid 2(\mathrm{~N}+\mathrm{n}))}{\mathrm{OSP}(2 \mathrm{n} \mid 2 \mathrm{n}) \times \operatorname{OSP}(2 \mathrm{~N} \mid 2 \mathrm{~N})}
$$

are promising candidates for conformal sigma models for all non negative values of $N$ and $n$.

- If we specialize to $M=n=2, m=N=0$ and change the reality conditions appropriately, we obtain the well known $\mathbb{Z}_{4}$ coset space PSU $(2,2 \mid 4) / \mathrm{SO}(4,1) \times \mathrm{SO}(5)$ whose bosonic base is $A d S_{5} \times S^{5}$. This model cannot be reduced any further, since $\mathfrak{g}^{\prime}$ is purely bosonic. It constitutes the maximal reduction of the two parameter discrete family of models

$$
\mathcal{M}_{(m, n)}=\frac{\operatorname{PSU}(2 m+2 n+2,2 \mid 2 m+2 n+4)}{\operatorname{OSP}(2 \mathrm{~m} \mid 2 \mathrm{~m}+2,2) \times \operatorname{OSP}(2 \mathrm{n} \mid 2 \mathrm{n}+4)} .
$$

- Setting $M=n=0, m=N=1$ and again taking the appropriate boundary conditions, leads to the space PSU $(1,1 \mid 2) / \mathrm{SO}(2) \times \mathrm{SO}(2)$ whose bosonic base is $A d S_{2} \times S^{2}$. This case is the maximal reduction of the family of sigma models with $\mathfrak{g}=\operatorname{psu}(2(m+n+1) \mid 2(m+n+1))$ and $\mathfrak{g}^{\prime}=\operatorname{osp}(2 m+2 \mid 2 m) \oplus \operatorname{osp}(2 n+2 \mid 2 n)$, subject to a certain reality conditions.

Example 2. We are interested in the $\mathbb{Z}_{4}$ coset

$$
\mathfrak{g} / \mathfrak{g}^{\prime}=\frac{\operatorname{osp}(M+2 m \mid 2 N+2 n)}{\operatorname{osp}(p \mid 2 q) \oplus \operatorname{osp}(M-p \mid 2(N-q)) \oplus \mathrm{u}(m \mid n)} .
$$


The corresponding automorphism is $\Omega=A d_{X}$ with

$$
X=\left(\begin{array}{ll|lll}
I_{M}^{p} & & & \\
& J_{2 m} & & & \\
\hline & I_{N}^{q} & & \\
& & I_{N}^{q} & \\
& & & J_{2 n}
\end{array}\right) \text { where } \begin{gathered}
I_{n}^{p}=\left(\begin{array}{cc}
\mathbb{1}_{p} & 0 \\
0 & -\mathbb{1}_{n-p}
\end{array}\right) \\
J_{2 n}=\left(\begin{array}{cc}
0 & \mathbb{1}_{n} \\
-\mathbb{1}_{n} & 0
\end{array}\right) .
\end{gathered}
$$

Under the action of $\mathfrak{g}^{\prime}$, the $\Omega$ eigenspaces transform as

$$
\begin{aligned}
& \mathfrak{m}_{1} \cong(\square \otimes \varnothing \otimes \square) \oplus(\varnothing \otimes \square \otimes \square) \\
& \mathfrak{m}_{2} \cong(\varnothing \otimes \varnothing \otimes \square) \oplus\left(\varnothing \otimes \varnothing \otimes \square^{*}\right) \oplus(\square \otimes \square \otimes \varnothing) \\
& \mathfrak{m}_{3} \cong\left(\square \otimes \varnothing \otimes \square \square^{*}\right) \oplus\left(\varnothing \otimes \square \otimes \square^{*}\right),
\end{aligned}
$$

where by $U \otimes V \otimes W$ we understand a module defined as the tensor product of the $U, V, W$ representations of respectively osp $(p \mid 2 q)$, osp $(M-p \mid 2(N-q))$ and $\mathrm{u}(m \mid n)$. When selecting the fermionic operator $Q \in \mathfrak{g}^{\prime}$, we choose it to be fully contained in one of the direct summands of $\mathfrak{g}^{\prime}$. Since the first two lead, after suitable choice of the parameters $M, N, p, q$, to the same reduction, we will assume, that $Q$ is either in $\operatorname{osp}(M-p \mid 2(N-q))$ or in $\mathrm{u}(m \mid n)$.

- If now we have $M=2 N$ and $p=2 q$, then we can pursue the reduction of the first type until we get rid of the orthosymplectic parts in $\mathfrak{g}^{\prime}$ to arrive at the sigma model on the symmetric space $\operatorname{OSP}(2 \mathrm{~m} \mid 2 \mathrm{n}) / \mathrm{U}(\mathrm{m} \mid \mathrm{n})$ which is not a conformal theory.

- If on the other hand $m=n$, then taking the second type of reduction can be used to remove the unitary part of $\mathfrak{g}^{\prime}$ so as to obtain the sigma model on the symmetric space $\operatorname{OSP}(\mathrm{M} \mid 2 \mathrm{~N}) / \operatorname{OSP}(\mathrm{p} \mid 2 \mathrm{q}) \times \operatorname{OSP}(\mathrm{M}-\mathrm{p} \mid 2(\mathrm{~N}-\mathrm{q}))$, which is a conformal field theory for $p=1, q=0$ and $M=2 N+2$. We therefore come to the conclusion that for all $N, n \in \mathbb{N}$ the sigma models on the homogeneous spaces

$$
\mathcal{C}_{(N, n)} \cong \frac{\operatorname{OSP}(2 \mathrm{~N}+2+2 \mathrm{n} \mid 2 \mathrm{~N}+2 \mathrm{n})}{\operatorname{OSP}(2 \mathrm{~N}+1 \mid 2 \mathrm{~N}) \times \mathrm{U}(\mathrm{n} \mid \mathrm{n})}
$$

are candidates for conformally invariant sigma models. For $n=0$ they reduce to the symmetric spaces $S^{2 N+1 \mid 2 N}$, i.e. the superspheres, whereas for $N=0$ they remain a $\mathbb{Z}_{4}$ homogeneous space.

Example 3. The last case under consideration is the $\mathbb{Z}_{4}$ coset

$$
\mathfrak{g} / \mathfrak{g}^{\prime}=\frac{\mathrm{u}(M+2 m \mid N+2 n)}{\mathrm{u}(p \mid q) \oplus \mathrm{u}(M-p \mid N-q) \oplus \mathrm{u}(m \mid n) \oplus \mathrm{u}(m \mid n)}
$$

defined by the automorphism $\Omega=A d_{Y}$, where

$$
Y=\left(\begin{array}{ll|ll}
I_{M}^{p} & & & \\
& J_{2 m} & & \\
\hline & & I_{N}^{q} & \\
& & & J_{2 n}
\end{array}\right) .
$$


We need not spell out the decomposition of $\mathfrak{m}_{i}$ in modules of $\mathfrak{g}^{\prime}$, it suffices to say that the only representations that appear are of the kind $A \otimes B \otimes C \otimes D$, where $A, B, C, D$ are either the trivial, fundamental or dual fundamental of respectively $\mathrm{u}(p \mid q), \mathrm{u}(M-p \mid N-q)$, the first $\mathrm{u}(m \mid n)$ and the second $\mathrm{u}(m \mid n)$. We choose $Q$ to be diagonally embedded in the $\mathrm{u}(m \mid n) \oplus \mathrm{u}(m \mid n)$ part of $\mathfrak{g}^{\prime}$, so that the reduction procedure sends the parameters $m$ and $n$ to $m-1$ and $n-1$. If $m=n$, then the reduction terminates with the symmetric space $\mathrm{U}(\mathrm{M} \mid \mathrm{N}) / \mathrm{U}(\mathrm{p} \mid \mathrm{q}) \times \mathrm{U}(\mathrm{M}-\mathrm{p} \mid \mathrm{N}-\mathrm{q})$. The sigma models with this target spaces are conformal for $M=N$ and $p=q \pm 1$, with the special case $p=1$ and $q=0$ corresponds to the complex symmetric superspaces $\mathbb{C P}^{\mathrm{N}-1 \mid \mathrm{N}}$. In conclusion, we can state that the sigma models on the homogeneous spaces

$$
\mathcal{C}_{(M, N, n)} \cong \frac{\mathrm{U}(\mathrm{M}+\mathrm{N}+2 \mathrm{n} \mid \mathrm{M}+\mathrm{N}+2 \mathrm{n})}{\mathrm{U}(\mathrm{M}+1 \mid \mathrm{M}) \times \mathrm{U}(\mathrm{N}-1 \mid \mathrm{N}) \times \mathrm{U}(\mathrm{n} \mid \mathrm{n}) \times \mathrm{U}(\mathrm{n} \mid \mathrm{n})},
$$

are expected to be conformal for values of $M, N, n \in \mathbb{N}$ with $N>0$.

\subsection{Extensions of the cohomological reduction}

In this section, we want to expand the technique of cohomological reduction to encompass Wess-Zumino-Witten and Gross-Neveu models.

The Wess-Zumino term on the supergroup $G$ with the superalgebra $\mathfrak{g}$ takes the form

$$
\begin{aligned}
\mathcal{S}_{\mathrm{WZ}} & =-\frac{i}{24 \pi} \int_{B}\left(g^{-1} \mathrm{~d} g,\left[g^{-1} \mathrm{~d} g, g^{-1} \mathrm{~d} g\right]\right) \\
& =-\frac{i}{24 \pi} \int_{B} d^{3} x \epsilon^{\alpha \beta \gamma} f_{a b c} J_{\alpha}^{a} J_{\beta}^{b} J_{\gamma}^{c},
\end{aligned}
$$

where $\epsilon^{\alpha \beta \gamma}$ is the antisymmetric tensor and $f_{a b c}$ are the structure constants. Thus, the Wess-Zumino term is a trilinear combination of the left invariant currents $J$ and can be cohomologically reduced in a similar fashion as the bilinear $\mathrm{G}$ and $\mathrm{B}$ terms.

A straight forward if lengthy computation shows that, choosing a fermionic operator $Q \in \mathfrak{g}$ that squares to zero, the cohomologically reduced model is the Wess-Zumino-Witten model on the supergroup $H$ whose superalgebra is $\mathrm{H}_{Q}(\mathfrak{g})$. Thus, the Wess-Zumino-Witten models on a supergroup reduce in the same way as the principal chiral models on the same supergroup. It can even happen that a principal chiral model and a WZW model both reduce to the same theory. An interesting example of that is furnished by the $\operatorname{PSU}(\mathrm{N} \mid \mathrm{N})$ WZW and principal chiral, the maximal reduction of which is the $c=-2$ free theory of a single pair of symplectic fermions.

Let us now turn to a very different theory, namely the $\operatorname{osp}(m \mid 2 n)$ Gross Neveu model for $m$ free real fermions and $n$ pairs of bosonic ghosts. The free part of the action is determined through

$$
\mathcal{S}_{\text {free }}^{\mathrm{GN}}=\frac{1}{2 \pi} \int_{\Sigma} d^{2} z\left[\sum_{i=1}^{m}\left(\psi_{i} \bar{\partial} \psi_{i}+\bar{\psi}_{i} \partial \bar{\psi}_{i}\right)+\sum_{a=1}^{n}\left(\beta_{a} \bar{\partial} \gamma_{a}+\bar{\beta}_{a} \partial \bar{\gamma}_{a}\right)\right] .
$$

This action defines a conformal field theory with central charge $c=\frac{m-2 n}{2}$ and both left and right $\widehat{\mathrm{osp}}(m \mid 2 n)$ current symmetry at level $k=1$. The conformal dimension of the 
fundamental fields $\left(\psi_{i}, \beta_{a}, \gamma_{b}\right)$ is $h=\frac{1}{2}$. The interaction term for this theory is

$$
\mathcal{S}_{\mathrm{int}}^{\mathrm{GN}}=\frac{g^{2}}{2 \pi} \int_{\Sigma} d^{2} z\left[\sum_{i=1}^{m} \psi_{i} \bar{\psi}_{i}+\sum_{a=1}^{n}\left(\gamma_{a} \bar{\beta}_{a}-\beta_{a} \bar{\gamma}_{a}\right)\right]^{2} .
$$

An alternative way of understanding this model, is to think of the free part of the theory as a free field representation of the Wess-Zumino-Witten model of OSP $(2 \mathrm{~m} \mid 2 \mathrm{n})$ at level one and of the interacting model as being a current-current perturbation thereof.

If at the free point, we take the fermionic nilpotent generator

$$
Q=\frac{1}{4 \pi i}\left\{\oint_{0} d z\left(\psi_{1}+i \psi_{2}\right) \beta_{1}-\oint_{0} d \bar{z}\left(\bar{\psi}_{1}+i \bar{\psi}_{2}\right) \bar{\beta}_{1}\right\}
$$

we see that

$$
\mathcal{L}_{\mathrm{int}}^{\mathrm{GN}}=\left[\sum_{i=3}^{m} \psi_{i} \bar{\psi}_{i}+\sum_{a=2}^{n}\left(\gamma_{a} \bar{\beta}_{a}-\beta_{a} \bar{\gamma}_{a}\right)\right]^{2}+Q \cdot B .
$$

It is furthermore not hard to see that a field is in the cohomology of $Q$ if and only if it does not contain any contribution from the fields $\psi_{1}, \psi_{2}, \beta_{1}, \gamma_{1}$. The cohomologically reduced model is therefore the $\operatorname{osp}(m-2 \mid 2(n-1))$ Gross Neveu model. An interesting example is obtained if we set $m=2 n+2$, in which case the maximal reduction of the Gross Neveu model is the massless Thirring model of two real fermions, which is dual to the theory of a compactified free boson. On the other hand, the compactified free boson provides the endpoint of the cohomological reduction of the sigma models on the superspheres $S^{2 n+1 \mid 2 n}$. In $[16,17]$, it was proposed that there exists a duality between the $\operatorname{osp}(2 n+2 \mid 2 n)$ Gross Neveu models and the sigma models on $S^{2 n+1 \mid 2 n}$ and what we see here supports this claim.

\section{Conclusion and outlook}

In this paper we have studied correlation functions of quantum field theories with internal supersymmetry. Most of the analysis was tailored towards 2-dimensional sigma models on coset superspaces $G / G^{\prime}$. For such theories we chose a BRST operator $Q \in \mathfrak{g}^{\prime}$ and performed a cohomological reductions to another coset sigma model $H / H^{\prime}$. Fields of the latter were shown to be in one-to-one correspondence with the cohomology $\mathrm{H}_{Q}\left(\mathcal{H}^{\mathcal{G}} / \mathcal{G}^{\prime}\right)$ in the state space $\mathcal{H}^{\mathcal{G}} / \mathcal{G}^{\prime}$ of the $G / G^{\prime}$ model. This correspondence preserves all correlators. Let us stress once more that reductions of this type are certainly not restricted to sigma models. Similar arguments also apply to non-geometric theories such as e.g. Gross-Neveu or Landau-Ginsburg models.

Before we conclude, let us sketch a number of possible applications. The results of this work have been used already in [18] for an investigation of boundary spectra in sigma models on complex projective superspaces $\mathbb{C P}^{\mathrm{N}-1 \mid \mathrm{N}}$. For vanishing coupling, it is an easy combinatorial exercise to determine the spectra of these sigma models. Our strategy then was to calculate the spectrum at finite coupling by assuming so-called Casimir evolution of conformal weights $[16,31]$. This assumption was tested carefully both through background field expansions and extensive numerical studies. From the spectrum at vanishing coupling 
along with the assumed Casimir evolution, the boundary partition functions can be constructed up to two unknown functions. This is were the cohomological reduction comes in. In fact, we then showed that the two unknown functions may be identified as conformal weights of fields belonging to the symplectic fermion subsector of the $\mathbb{C P}^{\mathrm{N}-1 \mid \mathrm{N}}$ coset model. Since symplectic fermions are free, we could fix all the remaining freedom and find an exact analytic expression for boundary partition functions of the $\mathbb{C P}^{\mathrm{N}-1 \mid \mathrm{N}}$ sigma model.

Another application concerns the issue of conformal symmetry. For sigma models on symmetric superspaces $G / G^{\mathbb{Z}_{2}}$ we found a free subsector $H / H^{\mathbb{Z}_{2}}$ if and only if the original model was conformal. We also provided several examples of more general coset superspaces $G / G^{\prime}$ that possess a free subsector. Though we are not prepared to argue that the corresponding $G / G^{\prime}$ coset sigma models are in fact conformal, we believe that this is the case, at least for an appropriate choice of the background fields G and B. In any case, the issue certainly deserves further investigation.

As we have stated in the introduction, one of the main motivations for the study of superspace sigma models comes from the AdS/CFT correspondence. It is likely that the ideas of this work can be adjusted so as to apply to models that are relevant for the study of strings in AdS geometries. In the case of $A d S_{3}$, for example, correlation functions of chiral primaries have been computed in the NSR formalism using the explicit solution of the WZNW model on the bosonic space $H_{3}^{+} \times \mathrm{SU}(2)$. A closer look at the results of $[32,33]$ shows that most of the intricate features of the full WZNW model cancel out from the correlation function of chiral primaries. The answer looks much simpler than one might naively expect, very much like a three point function in some free field theory. We hope to re-derive and extend these findings through a cohomological reduction, after re-phrasing the computation in the target space supersymmetric hybrid formalism [11]. A detailed analysis in currently being pursued.

Concerning the study strings of $A d S_{5}$, concrete applications seem a little more speculative. Within the pure spinor approach, strings in $A d S_{5} \times S^{5}$ may be described by coupling some superspace coset model $G / G^{\mathbb{Z}_{4}}$ to the pure spinor ghost sector [2]. The coset model is one of the examples we described in section 5. Since its denominator group is purely bosonic, we cannot apply our ideas, neither to the matter sector alone nor to the full theory. Actually, in this case any element $Q \in \operatorname{psl}(N \mid N)$ from the numerator Lie superalgebra satisfying $Q^{2}=0$ may be shown to possess trivial cohomology. A non-trivial subsector can only emerge after restricting to physical states, i.e. to the cohomology of the world-sheet BRST operator $Q_{\mathrm{BRST}}$ of the pure spinor theory. Generalizing our work to this setup would require a thorough analysis of the bi-complex that is generated by the BRST operator $Q_{\mathrm{BRST}}$ along with a space-time supersymmetry $Q$. A more direct application of the cohomological reduction to strings in $A d S_{5}$ might be possible within the light-cone gauge fixed Green-Schwarz formulation (see e.g. [5]). In this approach, the unbroken space-time symmetries are described by two copies of a centrally extended psu $(2 \mid 2)$ algebra which share their central elements. It might be feasible to use some of the corresponding fermionic generators for a cohomological reduction. It seems interesting to explore such cohomological reductions for string theory in $A d S_{5}$ backgrounds. 
Open Access. This article is distributed under the terms of the Creative Commons Attribution Noncommercial License which permits any noncommercial use, distribution, and reproduction in any medium, provided the original author(s) and source are credited.

\section{References}

[1] R.R. Metsaev and A.A. Tseytlin, Type IIB superstring action in $A d S_{5} \times S^{5}$ background, Nucl. Phys. B 533 (1998) 109 [hep-th/9805028] [SPIRES].

[2] N. Berkovits, Super-Poincaré covariant quantization of the superstring, JHEP 04 (2000) 018 [hep-th/0001035] [SPIRES].

[3] B. Stefanski jr, Green-Schwarz action for type IIA strings on $A d S_{4} \times C P^{3}$, Nucl. Phys. B 808 (2009) 80 [arXiv: 0806.4948] [SPIRES].

[4] P. Fré and P.A. Grassi, Pure spinor formalism for OSP (N|4) backgrounds, arXiv:0807.0044 [SPIRES].

[5] G. Arutyunov and S. Frolov, Foundations of the $A d S_{5} \times S^{5}$ superstring. Part I, J. Phys. A 42 (2009) 254003 [arXiv:0901.4937] [SPIRES].

[6] G. Arutyunov and S. Frolov, Superstrings on $A d S_{4} \times \mathbb{C P}^{3}$ as a coset $\sigma$-model, JHEP 09 (2008) 129 [arXiv: 0806 .4940] [SPIRES].

[7] N. Read and H. Saleur, Enlarged symmetry algebras of spin chains, loop models and S-matrices, Nucl. Phys. B 777 (2007) 263 [cond-mat/0701259] [SPIRES].

[8] C. Candu, J.L. Jacobsen, N. Read and H. Saleur, Universality classes of dense polymers and conformal $\sigma$-models, arXiv:0908.1081 [SPIRES].

[9] M.R. Zirnbauer, Conformal field theory of the integer quantum Hall plateau transition, hep-th/9905054 [SPIRES].

[10] P.B. Wiegmann, Superconductivity in strongly correlated electronic systems and confinement versus deconfinement phenomenon, Phys. Rev. Lett. 60 (1988) 821 [SPIRES].

[11] N. Berkovits, C. Vafa and E. Witten, Conformal field theory of AdS background with Ramond-Ramond flux, JHEP 03 (1999) 018 [hep-th/9902098] [SPIRES].

[12] M. Bershadsky, S. Zhukov and A. Vaintrob, $P S L(n \mid n) n \sigma$-model as a conformal field theory, Nucl. Phys. B 559 (1999) 205 [hep-th/9902180] [SPIRES].

[13] N. Read and H. Saleur, Exact spectra of conformal supersymmetric nonlinear $\sigma$-models in two dimensions, Nucl. Phys. B 613 (2001) 409 [hep-th/0106124] [SPIRES].

[14] A. Babichenko, Conformal invariance and quantum integrability of $\sigma$-models on symmetric superspaces, Phys. Lett. B 648 (2007) 254 [hep-th/0611214] [SPIRES].

[15] C. Candu and H. Saleur, A lattice approach to the conformal OSP $(2 S+2 \mid 2 S)$ supercoset $\sigma$-model. Part I: algebraic structures in the spin chain. The Brauer algebra, Nucl. Phys. B 808 (2009) 441 [arXiv:0801.0430] [SPIRES].

[16] C. Candu and H. Saleur, A lattice approach to the conformal OSP (2S+2|2S) supercoset $\sigma$-model. Part II: the boundary spectrum, Nucl. Phys. B 808 (2009) 487 [arXiv:0801. 0444] [SPIRES].

[17] V. Mitev, T. Quella and V. Schomerus, Principal chiral model on superspheres, JHEP 11 (2008) 086 [arXiv:0809.1046] [SPIRES]. 
[18] C. Candu, V. Mitev, T. Quella, H. Saleur and V. Schomerus, The $\sigma$-model on complex projective superspaces, JHEP 02 (2010) 015 [arXiv:0908.0878] [SPIRES].

[19] J.M.F. Labastida and C. Lozano, Lectures on topological quantum field theory, hep-th/9709192 [SPIRES].

[20] S. Helgason, Differential geometry, Lie groups and symmetric spaces, American Mathematical Society, U.S.A. (2001).

[21] S. Sethi, Supermanifolds, rigid manifolds and mirror symmetry, Nucl. Phys. B 430 (1994) 31 [hep-th/9404186] [SPIRES].

[22] C. Candu, Discrétisation des modèles sigma invariants conformes sur des supersphères et des superespaces projectifs (in French), Ph.D. thesis, Université Paris 6, Paris France (2008).

[23] N. Berkovits, M. Bershadsky, T. Hauer, S. Zhukov and B. Zwiebach, Superstring theory on $A d S_{2} \times S^{2}$ as a coset supermanifold, Nucl. Phys. B 567 (2000) 61 [hep-th/9907200] [SPIRES].

[24] D. Kagan and C.A.S. Young, Conformal $\sigma$-models on supercoset targets, Nucl. Phys. B $\mathbf{7 4 5}$ (2006) 109 [hep-th/0512250] [SPIRES].

[25] C.A.S. Young, Non-local charges, $\mathbb{Z}_{m}$ gradings and coset space actions, Phys. Lett. B 632 (2006) 559 [hep-th/0503008] [SPIRES].

[26] M. Reed and B. Simon, Functional analysis, Academic Press, New York U.S.A. (1980).

[27] L. Frappat, P. Sorba and A. Sciarrino, Dictionary on Lie superalgebras, Academic Press Inc., San Diego U.S.A. (2000) [hep-th/9607161] [SPIRES].

[28] J. Germoni, Représentations indécomposables des superalgèbres de Lie spéciales linéaires (in French), Ph.D. thesis, Université Louis Pasteur, Strasbourg France (1997).

[29] J. Germoni, Indecomposable representations of osp $(3 \mid 2), D(2,1 \alpha)$ and $G(3)$, in Colloquium on Homology and Representation Theory (Spanish), Vaquerías Mexico (1998) [Bol. Acad. Nac. Cienc. (Córdoba) 65 (2000) 147].

[30] J. Jost, Riemannian geometry and geometric analysis, Springer, Germany (2002).

[31] T. Quella, V. Schomerus and T. Creutzig, Boundary spectra in superspace $\sigma$-models, JHEP 10 (2008) 024 [arXiv:0712.3549] [SPIRES].

[32] M.R. Gaberdiel and I. Kirsch, Worldsheet correlators in AdS $S_{3} / C F T_{2}, J H E P 04$ (2007) 050 [hep-th/0703001] [SPIRES].

[33] A. Pakman and A. Sever, Exact $N=4$ correlators of $A d S_{3} / C F T_{2}$, Phys. Lett. B 652 (2007) 60 [arXiv: 0704.3040] [SPIRES]. 